\title{
Elastic wave transmission at an abrupt junction in a thin plate with application to heat transport and vibrations in mesoscopic systems
}

\author{
M. C. Cross \\ Condensed Matter Physics 114-36, California Institute of Technology, Pasadena, California 91125 \\ Ron Lifshitz \\ School of Physics and Astronomy, Raymond Sackler Faculty of Exact Sciences, Tel Aviv University, Tel Aviv 69978, Israel
}

(Received 23 November 2000; published 8 August 2001)

\begin{abstract}
The transmission coefficient for vibrational waves crossing an abrupt junction between two thin elastic plates of different widths is calculated. These calculations are relevant to ballistic phonon thermal transport at low temperatures in mesoscopic systems and the $Q$ for vibrations in mesoscopic oscillators. Complete results are derived in a simple scalar model of the elastic waves, and results for long-wavelength modes are obtained using full elasticity theory. We suggest that thin-plate elasticity theory provides a useful and tractable approximation to the three-dimensional geometry.
\end{abstract}

DOI: 10.1103/PhysRevB.64.085324

PACS number(s): $62.30 .+\mathrm{d}, 63.22 .+\mathrm{m}, 66.70 .+\mathrm{f}$

\section{INTRODUCTION}

The electronic properties of mesoscopic systems have been studied experimentally and theoretically for many years. More recently the behavior of other excitations, for example, lattice vibrations (phonons) and spin degrees of freedom, have come under study in these systems. In this paper we present results relevant to the issues of heat transport by phonons and the dissipation of vibrational modes in mesoscopic systems.

The interest in heat transport by phonons in mesoscopic systems arises because for easily fabricated devices the wavelength of a typical thermal phonon becomes comparable to the dimension of the thermal pathway at accessible temperatures of order $1 \mathrm{~K}$. Thus quantized thermal transport due to the discrete mode structure of the thermal pathway should become evident. $\mathrm{We}^{1}$ and others ${ }^{2,3}$ showed that this leads to a natural quantum unit of thermal conductance $k_{B}^{2} T / h$ similar to the role of $e^{2} / h$ as a quantum of electrical conductance in one-dimensional wires. ${ }^{4}$ This quantum unit of thermal conductance is predicted $^{2}$ to be clearly observable at low enough temperatures where only the acoustic $(\omega \rightarrow 0)$ vibrational modes are excited in the thermal pathway-the waveguidelike modes with nonzero frequency cutoffs at long wavelengths are populated with exponentially small numbers: a universal thermal conductance is predicted equal to $N_{A} \pi^{2} k_{B}^{2} T / 3 h$ with $N_{A}$ the number of acoustic modes (four for a freely suspended beam of material, corresponding to a longitudinal mode, two bending modes, and a torsional mode). These predictions were recently verified in beautiful experiments by Schwab et al..$^{5}$

Vibrational modes in mesoscopic systems are found to have anomalously low- $Q$ values, compared to larger systems of the same material. ${ }^{6-11}$ At first sight, the dissipation might be expected to become smaller as the oscillator becomes smaller, since defects such as dislocations are eliminated when the size gets less than a typical defect separation. Thus the observation of lower values of $Q$ was a surprise. In addition, unexpected dependencies on temperature ${ }^{11}$ and mag- netic field ${ }^{7}$ have been observed and remain unexplained.

Both the possibility of observing the universal thermal conductance and explanations for the $Q$ of small resonators involve the properties of phonon excitations with a wavelength comparable to the system size. Here we investigate a particular issue relevant to both these questions, namely, the coupling of vibrational modes across an abrupt junction between two blocks of the same material but with different dimensions.

The geometry typical of a number of experiments is shown in Fig. 1. The "bridge" is made of silicon, silicon nitride, or gallium arsenide, is freely floating, and is of rectangular cross section. The bridge is connected to two larger blocks of the same semiconductor. In thermal-conduction experiments the block at one end, called the cavity, is also freely floating (and is physically supported by four bridges) and is of the same thickness. The block at the other end provides both the mechanical support and a thermal reservoir. In recent experiments ${ }^{12}$ the dimensions were thickness $t=200 \mathrm{~nm}$, width $b=300 \mathrm{~nm}$, and length $L=5 \mu \mathrm{m}$. In vibration experiments the bridge may be supported just at one end (a cantilever) ${ }^{13}$ or at both ends (a beam). ${ }^{9,11}$

An important question in both thermal transport and oscillator damping experiments is the coupling of the vibrational modes of the bridge to modes in the supports-how well the energy in a mode in the bridge is transmitted to the supports, and vice versa. We have previously introduced a

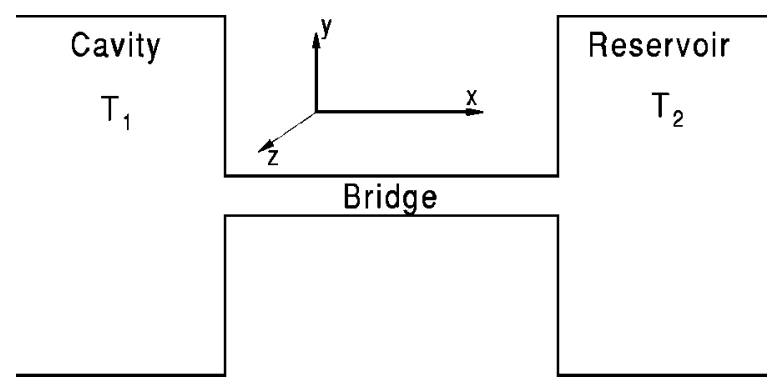

FIG. 1. Schematic of possible experimental geometry for the study of thermal transport and oscillations in mesoscopic systems. 
simple scalar model of the elastic waves to study this question in the context of thermal transport. ${ }^{1}$ In this paper we give a more realistic description of the vibrational modes.

First, in Sec. II, we introduce an improved scalar model, using a better choice for the boundary conditions on the scalar field that provides a more realistic approximation to the waves in an elastic medium. The scalar model in its revised form provides a useful first guide to the expected behavior of the experimental system, and a simpler environment in which to develop intuition and methods of theoretical attack. With the scalar model we perform a complete calculation of the scattering of the waves at the abrupt junction between the bridge and the supports for all the modes and at all wave vectors. We use the resulting transmission coefficients to evaluate the effect of the abrupt junction on the thermal conductance, particularly the universal low-temperature expression. In addition we introduce a simple method to calculate the transmission coefficients for the long-wavelength acoustic modes, and compare the results with the general results. In the full elastic calculation we will not be able to calculate the transmission coefficient for the full range of modes, but will be restricted to this type of long-wavelength calculation.

Second we propose that the elasticity theory for a thinplate geometry provides a useful semiquantitative description of the experimental geometry. This is described in Sec. III. The full elasticity theory for the modes in the twodimensional, thin-plate geometry is sufficiently tractable that a complete mode spectrum is readily calculated. On the other hand, a fully three-dimensional elasticity theory can only be attacked purely numerically. The two-dimensional theory reproduces many important features of a fully threedimensional elasticity theory, for example the mixing of bulk longitudinal and transverse waves by reflection at boundaries, the correct behavior of the dispersion relation at long wavelength and low frequency, including the "bending" modes with the unusual quadratic dispersion $\omega \propto k^{2}$ at long wavelengths, and regions of negative dispersion in the mode spectra. Thus the results should be more informative than the naive scalar model. The results should be accurate at sufficiently low temperatures where the modes with structure across the thickness are frozen out. We use the thin-plate model to investigate the mode structure in the beam, and the coupling of these modes to the supports, also treated as thin plates of the same thickness. Finally, in Sec. IV we apply the results to the issues of heat transport and oscillations in mesoscopic systems.

\section{A. Heat transport}

A thermal transport experiment is shown in Fig. 2. Two thermal masses are connected by four thermal pathways of mesoscopic dimensions in which heat transport by phonons is the dominant mechanism. One of the thermal masses, which we call the cavity, is a freely suspended thin block of semiconductor, with resistive wires on the upper surface to act as heat source and thermometer. The four bridges act as the thermal pathway to the outside world, as well as mechanical supports. Conceptually, heat is added to the cavity by resistive heating, and the resulting temperature difference

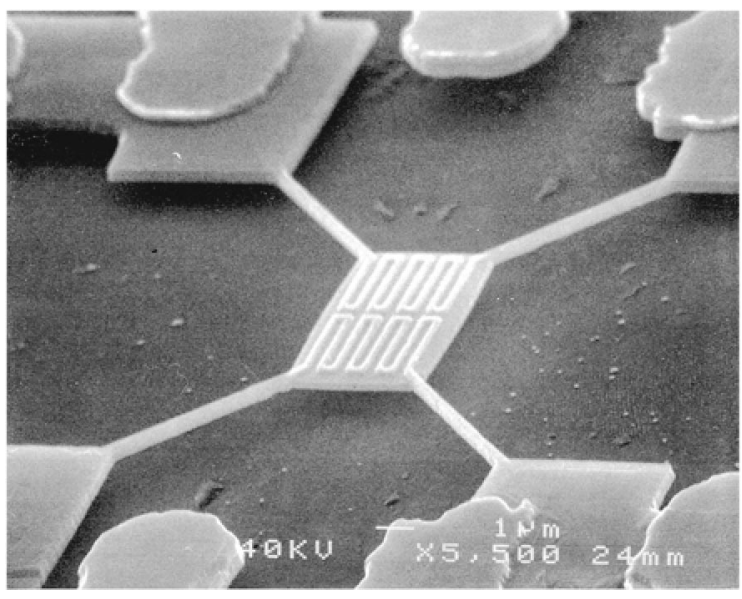

FIG. 2. Experimental geometry of Tighe et al. (Ref. 12).

from the reservoir is measured, yielding, for small heating, the thermal conductance of the bridges. In practice issues such as the thermal contact between the electrons in the resistive heater and thermometer and the phonons, and other thermal pathways to the reservoir such as through the electrical contacts to the resistive heaters, have to be considered. In this paper we will focus on the ideal situation where the phonon thermal pathway of the bridge dominates the conductance.

In mesoscopic systems it is easy to cool to temperatures where the transverse dimensions of the beam are comparable to or smaller than the typical phonon wavelength $h c / k_{B} T$ where $k_{B}$ is Boltzmann's constant, $h$ is Planck's constant, $c$ is a typical speed of sound in the material, and $T$ is the temperature. When this condition is satisfied the discrete mode structure of the thermal pathway becomes evident. Angelescu et al. ${ }^{1}$ showed that the thermal conductance takes on a quasiuniversal form, largely independent of the material and mode structure of the beam, on the assumption that the contact between the modes in the bridge and the cavity and reservoir can be considered ideal. An ideal contact implies that the right-going phonon modes in the bridge in Fig. 1 are populated with a thermal distribution at the temperature of the cavity, and the left-going modes at the temperature of the reservoir.

In a thermal-conductance measurement the cavity and reservoir are maintained at temperatures $T+\delta T$ and $T$ with temperature difference $\delta T$ small compared to their mean temperature. If we first look at the transport by the right-moving phonons, the energy flux is

$$
H^{(+)}=\frac{1}{2 \pi} \sum_{m} \int_{0}^{\infty} d k v_{g m}(k) \hbar \omega_{m}(k) n\left(\omega_{m}(k)\right),
$$

where $k$ is the wave vector along the bridge, $\omega_{m}(k)$ is the dispersion relation of the $m$ th discrete mode of the bridge, and $v_{g m}=d \omega_{m}(k) / d k$ is the group velocity. Transforming the integral to an integral over frequencies yields an expression for the heat transport by right-moving phonons 


$$
H^{(+)}=\frac{1}{2 \pi} \sum_{m} \int_{\omega_{m}}^{\infty} d \omega \hbar \omega_{m}(k) n\left(\omega_{m}(k)\right),
$$

where $\omega_{m}$ is the cutoff frequency of the $m$ th mode, i.e., the lowest frequency at which this mode propagates. (We have assumed the $m$ th mode propagates to arbitrarily large frequencies. If a particular mode only propagates over a finite band of frequencies, the upper limit of the integral will be replaced by $\omega_{m}^{\max }$.) The key simplification in this result is that the group-velocity factor is cancelled by the density of states in transforming from an integration over wave numbers to an integration over frequencies.

For ideal coupling to the reservoirs the distribution function $n\left(\omega_{m}(k)\right)$ for the right-moving phonons in Eq. (2) is evaluated as the Bose distribution at the cavity temperature $T+\delta T$. The thermal conductance is given by subtracting the analogous expression $H^{(-)}$for the left-moving phonons given by Eq. (2), but now with the distribution $n\left(\omega_{m}(k)\right)$ given by the Bose distribution at the reservoir temperature $T$

$$
K=\lim _{\delta T \rightarrow 0} \frac{H^{(+)}(T+\delta T)-H^{(-)}(T)}{\delta T} .
$$

Finally, introducing the scaled frequency variable $x$ $=\hbar \omega / k_{B} T$ gives the expression ${ }^{1}$

$$
K=\frac{k_{B}^{2} T}{h} \sum_{m} I\left(\hbar \omega_{m} / k_{B} T\right),
$$

where $I$ is given by the integral

$$
I(x)=\int_{x}^{\infty} \frac{y^{2} e^{y}}{\left(e^{y}-1\right)^{2}} d y .
$$

Equation (4) demonstrates the important result that the properties of the bridge only enter through the ratio of the mode cutoff frequencies to the temperature $\hbar \omega_{m} / k_{B} T$. The quantity $k_{B}^{2} T / h$ plays the role of the quantum unit of thermal conductance, analogous to the quantum of electrical conductance $e^{2} / h$ for one-dimensional wires. At very low temperatures the contribution to the thermal conductance by the modes with nonzero cutoff frequency will be exponentially small leaving a universal thermal conductance ${ }^{2}$

$$
K=N_{a} \frac{\pi^{2} k_{B}^{2} T}{3 h},
$$

where $N_{a}$ is the number of "acoustic" modes, i.e., modes with frequency tending to zero at long wavelengths. Usually this will be four for the beam (two transverse bending modes, one longitudinal compressional mode, and a torsional mode). Note that there is no dependence on the bridge properties in this expression.

More generally we cannot assume perfect coupling between the modes in the bridge and the cavity and reservoir. This can be taken into account, following the Landauer approach to electrical conductance, ${ }^{4}$ through a transmission coefficient for energy to be transported across the interfaces. For example for imperfect contact at the cavity-bridge inter- face we would find a thermal conductance (returning to unscaled quantities in the integral for clarity)

$$
K=\frac{\hbar^{2}}{k_{B} T^{2}} \sum_{m} \frac{1}{2 \pi} \int_{\omega_{m}}^{\infty} \mathcal{T}_{m}(\omega) \frac{\omega^{2} e^{\beta \hbar \omega}}{\left(e^{\beta \hbar \omega}-1\right)^{2}} d \omega,
$$

where $\mathcal{T}_{m}(\omega)$ is the energy transmission coefficient from the mode $m$ of the bridge at frequency $\omega$ into the cavity modes. Imperfect coupling at the bridge-reservoir junction, and elastic scattering due to imperfections in the bridge, can be similarly included through a total transmission matrix as in the electron case.

A central issue in predicting the thermal conductance is then to calculate the transmission coefficient $\mathcal{T}_{m}(\omega)$. This is particularly important in the question of the observability of the universal conductance at low temperatures, since the scattering of the long-wavelength phonons contributing to this quantity becomes strong-indeed for the abrupt junction in Fig. 1, $\mathcal{T}_{m}(\omega) \rightarrow 0$ as $\omega \rightarrow 0$ as we will see below. Although it is feasible in experiment to "smooth off" the corners, as indeed was done in the experiment of Schwab et al., consideration of the worst-case abrupt junction provides insight into the importance of geometric scattering.

\section{B. Oscillator $Q$}

The $Q$ of an oscillator is given by

$$
Q^{-1}=\frac{|\dot{E}|}{\omega E},
$$

where $\dot{E}$ is the rate of energy loss from the mode at frequency $\omega$ containing energy $E$. If we consider the oscillations of a beam supported by two supports, or a cantilever with one support, and estimate the energy loss as the energy transmitted into the supports, we find for the mode $n$

$$
Q_{n}^{-1} \sim \frac{v_{g}}{L \omega} \mathcal{T}_{n}
$$

where $v_{g}=d \omega / d k$ is the group velocity of a wave propagating in the beam, and $L$ is the length of the beam. The exact evaluation of this quantity depends on the nature of the mode (longitudinal, bending, etc.). For the longitudinal and torsional modes, which have a linear spectrum $\omega=c k$, the frequency of the fundamental mode in a beam of length $L$ is of order $c \pi / L$, the group velocity is $v_{g}=c$, and so

$$
Q_{n}^{-1} \sim \frac{\mathcal{T}_{n}}{n \pi} .
$$

For the bending waves with a quadratic spectrum $\omega \propto k^{2}$, the result is more complicated, but the $Q$ values are similar, and tend to this form for large $n$, so we will use this expression as a fairly accurate general estimate.

We see from Eq. (10) that good isolation of the mode $\mathcal{T}_{n}$ $\rightarrow 0$ is a criterion for high $Q$. In practice this expression for the dissipation may be an overestimate, since we are assuming that all the energy of the mode that enters the support either dissipates away, or propagates away to large distances 
so that the energy is not returned to the oscillations of the beam. If this is not the case, the transmission of energy into the support is only one part of the problem-we would also have to consider the behavior of the vibrational energy in the supports as well.

\section{SCALAR MODEL}

As a simple model of the elasticity problem consider a single scalar field $\phi$. This might represent, for example, the (scalar) "displacement," and the (vector) "stress" $\Sigma$ would then be proportional to $\nabla \phi$. We will suppose a twodimensional domain corresponding to the thin plate. This leads to a wave equation

$$
\frac{\partial^{2} \phi}{\partial t^{2}}=c^{2} \nabla^{2} \phi
$$

with $\nabla^{2}$ the two-dimensional Laplacian and $c$ giving the speed of propagation of the wave. Stress-free boundary conditions at the edges are then

$$
\hat{n} \cdot \nabla \phi=0
$$

with $\hat{n}$ the normal to the edge. Note that this Neumann boundary condition allows the propagation of an acoustic mode $[\omega(k \rightarrow 0) \rightarrow 0]$ in the bridge as we expect for elastic waves, whereas Dirichlet boundary conditions do not. An example of an elastic system described by such a scalar model is a stretched membrane: $\phi$ would then be the displacement normal to the membrane and $\hat{n} \cdot \Sigma$ is the vertical force on a unit line in the membrane normal to the direction $\hat{n}$.

The scalar problem is sufficiently simple that we can calculate the transmission across an abrupt junction such as the cavity-to-bridge junction in full detail. This allows us to gain insight into the more complicated elastic wave problem, and also allows us to illustrate and test approximation schemes that will be useful there.

The model, Eqs. (11) and (12), was studied by Angelescu et $a l .{ }^{1}$ using the mode matching method developed by Szafer and Stone ${ }^{14}$ for the analogous electron wave calculation. However, Angelescu et al. implicitly used a rather unnatural boundary condition $(\phi=0)$ for the end of the cavity at the junction plane [although Eq. (12) was assumed everywhere else, i.e., on the edges of the beam and cavity parallel to the propagation direction]. We briefly review this work, explain how this boundary condition was introduced, and then treat the more natural case [Eq. (12) everywhere] using the same methods. This new treatment actually removes a weak logarithmic divergence found in the original treatment, and produces results at low frequencies that are more consistent with the results of the full elasticity treatment.

\section{A. Model of Angelescu et al.}

Assume a simple two-dimensional geometry consisting of a rectangular bridge of transverse dimension $b$ connected to a rectangular cavity of transverse dimension $B$, Fig. 3 . In the

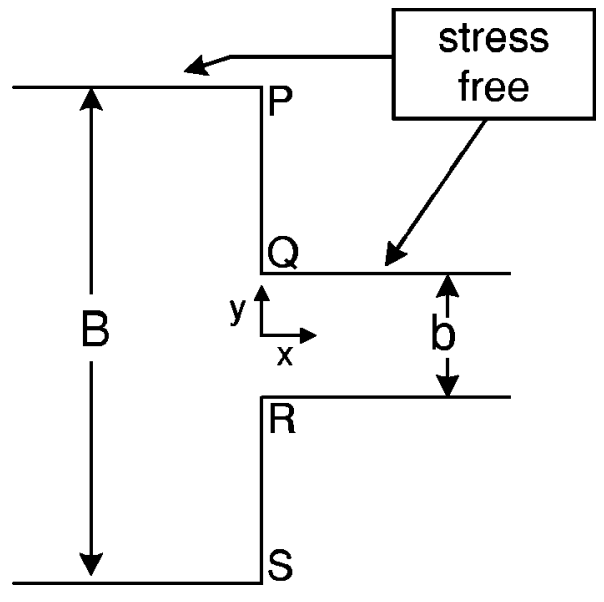

FIG. 3. Geometry for the calculation of the transmission coefficient. Stress-free boundary conditions are assumed on the edges as shown. Angelescu et al. used $\phi=0$ boundary conditions on the end $P Q$ and $R S$ of the cavity. A better choice is to use stress-free conditions here as well.

general three-dimensional case, if the cavity and bridge have the same thickness, there is no mixing of the $z$ modes, and the problem separates into a set of two-dimensional problems, one for each $z$ mode. Here we will only consider the lowest mode with no structure across the $z$ direction, which is the only mode excited at low enough temperatures. Let $\chi_{\alpha}^{c}(y)$ and $\chi_{m}(y)$ be orthonormal transverse modes satisfying the stress-free boundary conditions on the edges in the cavity and the bridge, respectively. (For clarity we will denote cavity-mode indices by Greek letters, and bridge-mode indices by Roman letters.) The solutions to the wave equation take the form

$$
\phi_{m}(x, y, t)=\chi_{m}(y) e^{i(k x-\omega t)}
$$

for the bridge, where the frequency of the mode $\omega$ is given by $\omega^{2}=\omega_{m}^{2}+c^{2} k^{2}$ with $\omega_{m}=m \pi c / b$ the cutoff frequency of the $m$ th bridge mode. The form is similar for the cavity modes with the cavity width $B$ replacing the bridge width $b$. We will denote the frequency separation between bridge modes by $\Delta$ :

$$
\Delta=\omega_{m+1}-\omega_{m}=\pi c / b .
$$

Consider a phonon incident on the interface from the cavity side $(x<0)$, in the mode $\alpha$ of the cavity, and with longitudinal wave vector $k_{\alpha}^{(c)}$. The solutions in the cavity and bridge, including the reflected waves in the cavity and the transmitted waves in the bridge, are

$$
\begin{gathered}
\phi^{(c)}=\chi_{\alpha}^{(c)} e^{i k_{\alpha}^{(c)} x}+\sum_{\beta} r_{\alpha \beta} \chi_{\beta}^{(c)} e^{-i k_{\beta}^{(c)} x} \quad \text { cavity, } \\
\phi=\sum_{m} t_{\alpha m} \chi_{m} e^{i k_{m} x} \quad \text { bridge }
\end{gathered}
$$

with $r_{\alpha \beta}$ and $t_{\alpha m}$ reflection and transmission amplitudes to be determined. In the above equations, $k_{m}$ and $k_{\beta}^{(c)}$ are the 
wave vectors of the transmitted and reflected waves, respectively, given by the frequency matching condition

$$
\omega^{2}=c^{2} k_{\alpha}^{(c) 2}+\omega_{\alpha}^{(c) 2}=c^{2} k_{m}^{2}+\omega_{m}^{2}=c^{2} k_{\beta}^{(c) 2}+\omega_{\beta}^{(c) 2} .
$$

Note that the sums over $m$ and $\beta$ in Eq. (15) include evanescent waves (imaginary $k$ or $k^{(c)}$ ) although only the propagating modes will contribute to the energy transport. The field $\phi$ and the longitudinal derivative $\partial \phi / \partial x$ have to be matched in the medium at $x=0$, which leads to the equations

$$
\begin{gathered}
\chi_{\alpha}^{(c)}+\sum_{\beta} r_{\alpha \beta} \chi_{\beta}^{(c)}=\sum_{m} t_{\alpha m} \chi_{m}, \\
k_{\alpha}^{(c)} \chi_{\alpha}^{(c)}-\sum_{\beta} r_{\alpha \beta} k_{\beta}^{(c)} \chi_{\beta}^{(c)}=\sum_{m} t_{\alpha m} k_{m} \chi_{m} .
\end{gathered}
$$

Equations for the reflection and transmission coefficients are extracted by integrating Eqs. (17) multiplied by a transverse function, $\chi_{m}$ or $\chi_{\alpha}^{(c)}$, and using the orthogonality of the functions over the appropriate domain to extract relationships for the mode coefficients. We first multiply one of the equations with a cavity mode $\chi_{\beta}^{(c)}$, and integrate over the cavity width, making use of the orthonormality relation $\int d y \chi_{\alpha}^{(c)} \chi_{\beta}^{(c)}=\delta_{\alpha \beta}$. In this section we follow Angelescu et al. ${ }^{1}$ and perform this operation on the first equation (i.e., the matching equation for the field $\phi$ ). It is at this stage that the boundary condition on the cavity field at the face $x=0$ for $|y|>b / 2$ is introduced. The replacement in the integration on the right-hand side

$$
\int_{-B / 2}^{B / 2} d y \chi_{\alpha}^{(c)} \phi(x=0) \Rightarrow \int_{b / 2}^{b / 2} d y \chi_{\alpha}^{(c)} \sum_{m} t_{\alpha m} \chi_{m}
$$

implicitly forces the boundary condition $\phi(x=0)=0$ for $|y|>b / 2$.

Multiplying the first equation in Eq. (17) by a cavity mode $\chi^{(c)}$, integrating over the cavity width, and using the orthonormality of the cavity modes leads to

$$
r_{\alpha \beta}=-\delta_{\alpha \beta}+\sum_{m} t_{\alpha m} a_{m \beta},
$$

where $a_{m \beta}$ is the overlap of cavity and bridge transverse functions

$$
a_{m \beta}=\int_{-b / 2}^{b / 2} d y \chi_{\beta}^{(c)} \chi_{m}
$$

Equation (19) may now be plugged into the second equation in Eq. (17), and the result is

$$
2 k_{\alpha}^{(c)} \chi_{\alpha}^{(c)}-\sum_{m} \sum_{\beta} t_{\alpha m} a_{m \beta} k_{\beta}^{(c)} \chi_{\beta}^{(c)}=\sum_{m} t_{\alpha m} k_{m} \chi_{m},
$$

which, when integrated with $\chi_{m}$ over the bridge width, yields

$$
2 k_{\alpha}^{(c)} a_{m \alpha}=\sum_{n} A_{n m} t_{\alpha n}+t_{\alpha m} k_{m} .
$$

This is a system of equations that determines $t_{\alpha m}$. In Eq. (22) the kernel $A_{m n}$ is given by

$$
A_{m n}=\sum_{\beta} a_{m \beta} a_{n \beta} k_{\beta}^{(c)} .
$$

These equations may be solved for the $t$ 's, and then the flux transmission probability from the wave-vector $k_{\alpha}^{(c)}$ state of cavity mode $\alpha$ to bridge mode $m$ is given by

$$
\mathcal{T}_{\alpha m}=\left|t_{\alpha m}\right|^{2} \frac{k_{m}}{k_{\alpha}^{(c)}} .
$$

Now summing over all the cavity modes that are propagating at frequency $\omega$ leads to the "transport transmission coefficient" from the cavity to the $m$ th mode $\mathcal{T}_{m}(\omega)$ (for $\omega$ $\left.>\omega_{m}\right)$ by

$$
\mathcal{T}_{m}(\omega)=\sum_{\alpha, \omega_{\alpha}^{(c)}<\omega} \mathcal{T}_{\alpha m}=\sum_{\alpha, \omega_{\alpha}^{(c)}<\omega}\left|t_{\alpha m}\right|^{2} \frac{k_{m}}{k_{\alpha}^{(c)}},
$$

with $k_{m}(\omega)$ and $k_{\alpha}^{(c)}(\omega)$ given by Eq. (16). This also gives the energy transmission coefficient from the $m$ th bridge mode to the cavity, by the usual reciprocity arguments.

Equations (23) and (25) involve sums over cavity modes. We may either evaluate the sums directly for a chosen value of the width ratio $B / b$, or take the limit of a large cavity width $B \rightarrow \infty$ when the sums are replaced by integrals. We calculate the matrix $A_{m n}$ for $m, n<N_{\max }$ with $N_{\max }$ some upper cutoff for the number of bridge modes retained and invert the $N_{\max } \times N_{\max }$ matrix system numerically to find $t_{\alpha m}$ and hence $\mathcal{T}_{m}(\omega)$.

There is a simple approximation ${ }^{14}$ that provides an analytic form for the solution to Eq. (22) that is in reasonably good agreement with the exact solution. The approximation derives from three properties of $a_{m \alpha}$. First, $a_{m \alpha}=0$ unless $m$ and $\alpha$ have the same parity. In other words, even modes couple to even modes and odd modes to odd modes only. Second, as a function of $\alpha, a_{m \alpha}$ is sharply peaked around $\alpha=m B / b$, the width of the peak being of order $B / b$. And third, $a_{\alpha m}$ must satisfy the completeness relation

$$
\sum_{\alpha} a_{m \alpha} a_{n \alpha}=\delta_{m n}
$$

The first two properties permit the key approximation, namely, that $A_{m n} \propto \delta_{m n}$ (since the product of two functions peaked at different channels $m, n$ is very small and $A_{m n}$ is rigorously zero when $m$ and $n$ are different parity modes). Then we only need the diagonal part of $A$

$$
A_{m m}=\sum_{\beta} a_{m \beta}^{2} k_{\beta}^{(c)},
$$

which is simply a weighted average of the complex wave vector over the narrow range of reflected cavity modes for 


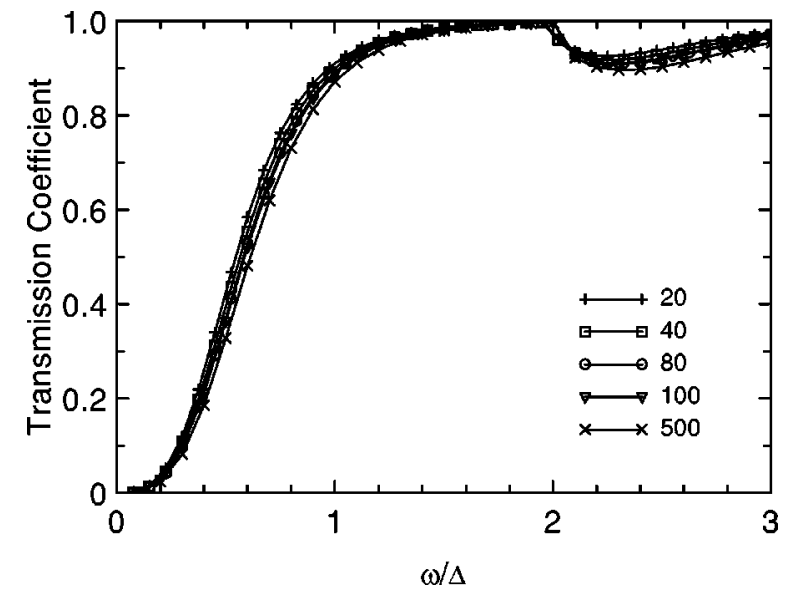

FIG. 4. Transmission coefficient $\mathcal{T}_{0}$ coupling the lowest bridge mode to the cavity modes as a function of the reduced frequency of the mode $\omega / \Delta$ with $\Delta$ the splitting between bridge modes at zero wave vector. Curves for cavity wave-number cutoff equal to 20,40 , 80,100 , and 500 times $\pi b^{-1}$ (with $b$ the width of the bridge) show the weak dependence on this cutoff.

which $a_{m \beta}$ is significant. (Note $\Sigma_{\beta} a_{m \beta}^{2}=1$ by completeness.) In this case Eq. (22) separates into

$$
2 k_{\alpha}^{(c)} a_{m \alpha}=A_{m m} t_{\alpha m}+k_{m} t_{\alpha m}
$$

and then

$$
t_{\alpha m}=\frac{2 k_{\alpha}^{(c)} a_{m \alpha}}{A_{m m}+k_{m}} .
$$

The flux transmission probability from cavity mode $\alpha$ to bridge mode $m$ is given in this approximation by

$$
\mathcal{T}_{\alpha m}=\left|t_{\alpha m}\right|^{2} \frac{k_{m}}{k_{\alpha}^{(c)}} \simeq \frac{4 k_{\alpha}^{(c)} k_{m}\left|a_{m \alpha}\right|^{2}}{\left(k_{m}+K_{m}\right)^{2}+J_{m}^{2}},
$$

where $K_{m}=\operatorname{Re} A_{m m}$ and $J_{m}=\operatorname{Im} A_{m m}$. The energy transmission coefficient is

$$
\mathcal{T}_{m}(\omega) \simeq \sum_{\alpha, \omega_{\alpha}^{(c)}<\omega} \frac{4 k_{\alpha}^{(c)} k_{m}\left|a_{m \alpha}\right|^{2}}{\left(k_{m}+K_{m}\right)^{2}+J_{m}^{2}}=\frac{4 K_{m} k_{m}}{\left(k_{m}+K_{m}\right)^{2}+J_{m}^{2}} .
$$

We now use the explicit form of the transverse modes to evaluate $\mathcal{T}_{m}(\omega)$. With the boundary condition $\partial \phi / \partial y=0$ at the $y$ boundaries we have

$$
\begin{aligned}
& \chi_{m}(y)= \begin{cases}\sqrt{\frac{2}{b} \cos \left(\frac{m \pi y}{b}\right)} & m \text { even, } \\
\sqrt{\frac{2}{b} \sin \left(\frac{m \pi y}{b}\right)} & m \text { odd, }\end{cases} \\
& \chi_{\alpha}^{c}(y)= \begin{cases}\sqrt{\frac{2}{B} \cos \left(\frac{\alpha \pi y}{B}\right)} & \alpha \text { even, } \\
\sqrt{\frac{2}{B} \sin \left(\frac{\alpha \pi y}{B}\right)} & \alpha \text { odd },\end{cases}
\end{aligned}
$$

with $m$ and $\alpha$ integers, with the special case $\chi_{0}(y)=1 / \sqrt{b}$ and $\chi_{0}^{c}(y)=1 / \sqrt{B}$. The $a_{m \alpha}$ are easily calculated:

$$
a_{m \alpha}=0 \quad m, \alpha \text { not both even or odd, }
$$

$$
\begin{aligned}
a_{m \alpha}= & \sqrt{\frac{b}{B}}\left[\frac{\sin \left(\frac{\alpha \pi b}{2 B}-\frac{m \pi}{2}\right)}{\frac{\alpha \pi b}{2 B}-\frac{m \pi}{2}}\right. \\
& \left.+\frac{\sin \left(\frac{\alpha \pi b}{2 B}+\frac{m \pi}{2}\right)}{\frac{\alpha \pi b}{2 B}+\frac{m \pi}{2}}\right] m, \alpha \text { even } \neq 0,
\end{aligned}
$$

$$
\begin{aligned}
a_{m \alpha}= & \sqrt{\frac{b}{B}}\left[\frac{\sin \left(\frac{\alpha \pi b}{2 B}-\frac{m \pi}{2}\right)}{\frac{\alpha \pi b}{2 B}-\frac{m \pi}{2}}\right. \\
& \left.-\frac{\sin \left(\frac{\alpha \pi b}{2 B}+\frac{m \pi}{2}\right)}{\frac{\alpha \pi b}{2 B}+\frac{m \pi}{2}}\right] m, \alpha \text { odd } \neq 0,
\end{aligned}
$$

$$
\begin{gathered}
a_{0 \alpha}=\sqrt{\frac{2 b}{B}}\left[\frac{\sin \left(\frac{\alpha \pi b}{2 B}\right)}{\frac{\alpha \pi b}{2 B}}\right] \alpha \text { even } \neq 0, \\
a_{m 0}=0 \quad m \neq 0, \\
a_{00}=1 .
\end{gathered}
$$

For large $m, \alpha$ (both even or both odd) we can approximate

$$
a_{m \alpha} \simeq \sqrt{\frac{b}{B}} \frac{\sin \left(\frac{\alpha \pi b}{2 B}-\frac{m \pi}{2}\right)}{\frac{\alpha \pi b}{2 B}-\frac{m \pi}{2}} .
$$

The $a_{m \alpha}$ are indeed sharply peaked as a function of cavitymode number $\alpha$. The large $m$ approximation is essentially identical to the result in the electronic case. ${ }^{14}$ However the small, second term in the braces in Eqs. (35) and (36) ignored in this approximation appears with the opposite sign in our application. This turns out to render the sum over the cavity modes $\beta$ appearing in Eq. (23) weakly (logarithmically) divergent for large $\beta$. (Note that $k_{\beta}^{(c)}$ is imaginary here, so this divergent contribution is to $\operatorname{Im} A_{m n}$ and to the compo- 


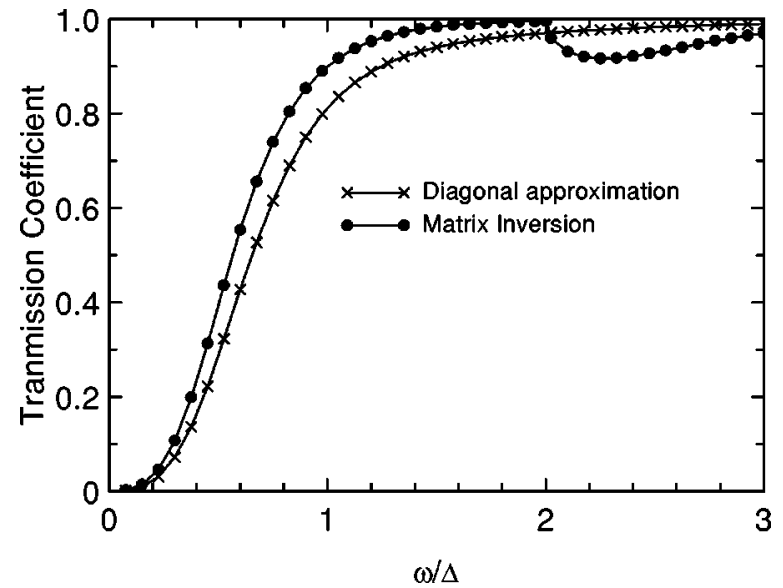

FIG. 5. Transmission coefficient $\mathcal{T}_{0}$ for the lowest bridge mode in the Angelescu et al. scalar model calculated using the (exact) matrix diagonalization (points) and the diagonal approximation (crosses) for a cavity-mode wave-vector cutoff $40 \pi b^{-1}$, with $b$ the width of the bridge. The diagonal approximation shows a somewhat stronger dependence on this cutoff than shown by the exact results, so that this comparison will vary as we change the cutoff assumption.

nent $J_{m}$ of the diagonal terms.) We must impose some upper cutoff to the sum to achieve finite results. Physically we might suppose such a cutoff may come from the breakdown of the sharp corner approximation at short enough scales, or ultimately, in a perfectly fabricated mesoscopic system, from the atomic nature of the material leading to a finite number of modes.

Results for the transmission coefficient of the lowest bridge mode $\mathcal{T}_{0}(\omega)$ are shown in Fig. 4 . There is only a weak dependence on the cavity sum cutoff. At small frequency $\mathcal{T}_{0}(\omega)$ is proportional to $\omega^{3}$. The results shown were calculated for the case of an infinitely wide cavity (sums over cavity modes replaced by integrals). Results for finite widths (e.g., $B / b=20$ ) are very similar. The first 11 bridge modes (six even modes) were retained in the matrix inversion for the results shown: increasing this number did not change the results significantly showing that $A_{m n}$ indeed decreases rapidly for increasing $|m-n|$. Note that $\mathcal{T}_{0}(\omega)$ rapidly approaches unity as the frequency grows. There is a small ( $\$ 10 \%$ ) decrease at the frequency $\omega=2 \Delta$ where the second even bridge mode becomes propagating, and similar features of reducing size occur at subsequent integral multiples of $2 \Delta$. There is no coupling between even and odd modes for the symmetric geometry used. The comparison between the results from the full matrix inversion and the diagonal approximation is shown in Fig. 5. The diagonal approximation gives results good to about $10 \%$. However this comparison depends on the cavity wave-number cutoff, since the diagonal approximation depends rather more strongly on this parameter than for the full results shown in Fig. 4. Note that the feature at $\omega=2 \Delta$ due to the interaction between different bridge modes is absent in the diagonal approximation.

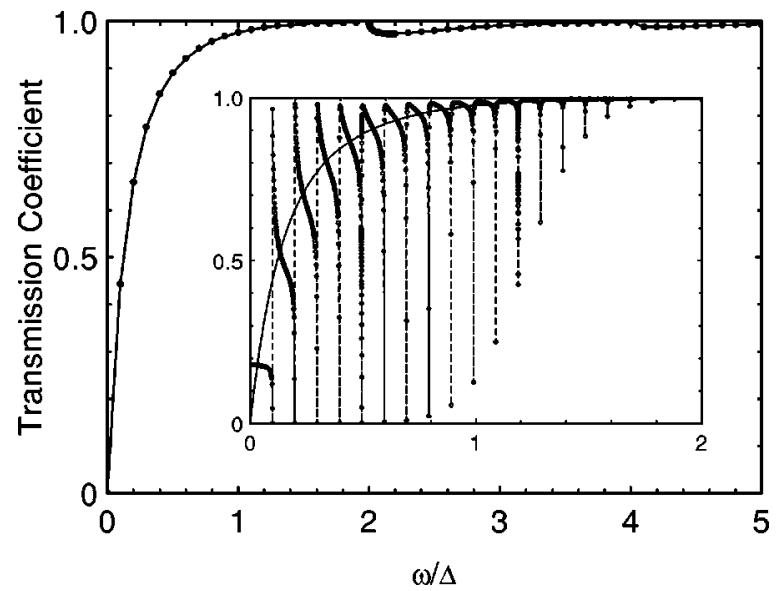

FIG. 6. Transmission coefficient $\mathcal{T}_{0}$ coupling the lowest bridge mode to the cavity modes as a function of the reduced frequency $\omega / \Delta$ in the scalar model with stress-free boundaries. The main graph is for an infinite cavity width. The inset shows the comparison with results for a finite cavity width $(B=20.217 b)$.

\section{B. Stress-free ends}

\section{Mode matching calculation}

We now redo the scalar analysis, enforcing the boundary condition $\hat{n} \cdot \nabla \phi=0$ on the end of the cavity $x=0,|y|$ $>b / 2$. This corresponds to a stress-free boundary everywhere.

The analysis proceeds as before up to Eq. (17). But now we first multiply the second equation (for the continuity of $\partial \phi / \partial x)$ by $\chi_{\beta}^{(c)}$, and integrate over the cavity width. This enforces the boundary condition on the cavity face

$$
\hat{n} \cdot \nabla \phi=0 \text { for } x=0 \text { and }|y|>b / 2 .
$$

The orthogonality of the $\chi_{\beta}^{(c)}$ gives

$$
r_{\alpha \beta} k_{\beta}^{(c)}=k_{\alpha}^{(c)} \delta_{\alpha \beta}-\sum_{m} t_{\alpha m} k_{m} a_{m \beta} .
$$

Use this equation to eliminate $r_{\alpha \beta}$ from the first of Eq. (17)

$$
2 \chi_{\alpha}^{(c)}-\sum_{n} \sum_{\beta} t_{\alpha n}\left(k_{n} / k_{\beta}^{(c)}\right) a_{n \beta} \chi_{\beta}^{(c)}=\sum_{m} t_{\alpha n} \chi_{n}
$$

and integrate with $\chi_{m}$ over the bridge width to yield

$$
2 a_{m \alpha}=\sum_{n} \bar{A}_{m n} k_{n} t_{\alpha n}+t_{\alpha m}
$$

where

$$
\bar{A}_{m n}=\sum_{\alpha} a_{m \alpha} a_{n \alpha} / k_{\alpha}^{(c)}
$$

Again we can solve the equation for $t_{\alpha m}$, Eq. (44), numerically or by using the diagonal approximation for $\bar{A}_{m n}$. It is easily seen that the extra inverse powers of $k_{\alpha}^{(c)}$ in $\bar{A}_{m n}$ render the sum over $\alpha$ convergent, unlike the case for $A_{m n}$. The 


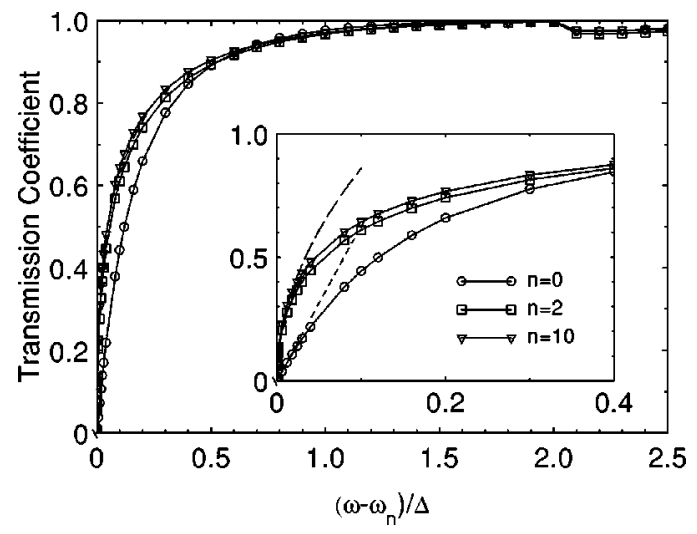

FIG. 7. Values of the transmission coefficients from the $n$th bridge mode to the cavity as a function of $\left(\omega-\omega_{n}\right) / \Delta$ with $\omega_{n}$ the cutoff frequency for the $n$th mode and $\Delta$ the spacing between mode cutoff frequencies $\Delta=\omega_{n+1}-\omega_{n}$. The long-dashed line shows the square-root dependence near cutoff for the $n \neq 0$ modes. The shortdashed line is the linear dependence $T_{0}(\omega) \simeq 2 \pi \omega / \Delta$ expected for the lowest mode.

energy transmission coefficient from the $m$ th bridge mode remains given by Eq. (25). The diagonal approximation $\bar{A}_{m n} \simeq \bar{A}_{m m} \delta_{m n}$ now leads to

$$
\mathcal{T}_{m}(\omega) \simeq \frac{4 \bar{K}_{m} k_{m}}{\left(k_{m} \bar{K}_{m}+1\right)^{2}+\bar{J}_{m}^{2} k_{m}^{2}},
$$

with $\bar{J}_{m}=\operatorname{Re} \bar{A}_{m m}$ and $\bar{K}_{m}=\operatorname{Im} \bar{A}_{m m}$. The result for $\mathcal{T}_{0}(\omega)$ for the lowest bridge mode is shown in Fig. 6 taking the limit of an infinite cavity width [the sums in Eq. (45), etc., evaluated as integrals]. Again the transmission coefficient grows rapidly, approaching close to unity as the frequency grows, for example reaching 0.9 by about $\omega \sim 0.5 \Delta$. Note the important difference from the previous scalar model that the lowfrequency asymptotic behavior is linear, $\mathcal{T}_{0}(\omega) \propto \omega$, rather than cubic as obtained there, and so $\mathcal{T}_{0}(\omega)$ approaches unity more rapidly than anticipated in that work. A small reduction in $\mathcal{T}_{0}(\omega)$ (by about 3\%) near $\omega=2 \Delta$ and by smaller amounts at higher integral multiples of this frequency are apparent. An analysis of the curve in this region shows a square-root dependence on $\omega-2 \Delta$, corresponding to a coupling in the full matrix calculation to the second bridge mode, and to the square-root growth in $\mathcal{T}_{2}(\omega)$ that occurs here. The diagonal approximation (not shown) gives results that are indistinguishable on the figure for $0 \leqslant \omega \leqslant 2 \Delta$, but the small decrease above $\omega=2 \Delta$ does not appear in this approximation.

The inset in Fig. 6 shows the comparison with results for a finite cavity width. The results are quite surprising, with resonancelike features occurring whenever the frequency passes through the cutoff frequency of a cavity mode. This can be traced to the inverse power of $k^{(c)}$ occurring in Eq. (25) and Eq. (45). Since $k^{(c)}$ goes to zero as $\sqrt{\omega-\omega_{n}^{(c)}}$ these singularities are integrable, and the features disappear in the limit of infinite cavity width. Smoothing over the features (e.g., taking the average over bins between successive $\omega_{n}^{(c)}$ ) gives points that follow the smooth curve for the infinite

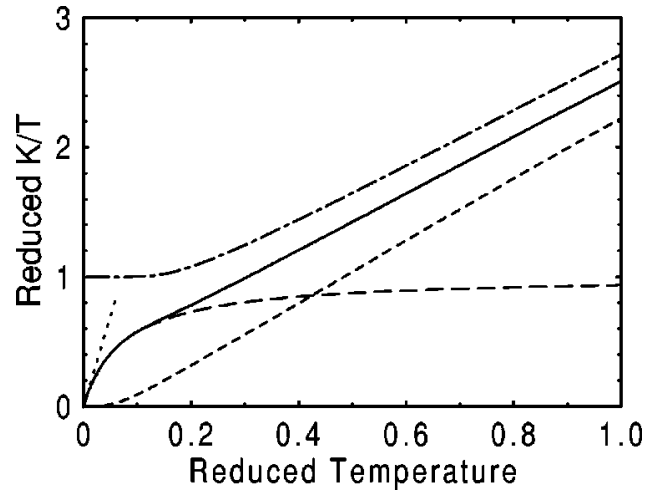

FIG. 8. Thermal conductivity divided by temperature reduced by the zero-temperature universal value $\pi^{2} k_{B}^{2} / 3 h$ as a function of the reduced temperature $k_{B} T / \hbar \Delta$ : solid curve-full calculation including the transmission losses due to the abrupt junction for stressfree face; long-dashed curve-contribution from the lowest (acoustic) mode, showing the reduction at low temperatures due to the scattering at the abrupt junction; dash-dotted curve-ideal result from all modes with full transmission; dotted line-lowtemperature asymptotic slope predicted from the low-frequency behavior of the transmission coefficient. The short-dashed curves shows the result including scattering of the calculation of Angelescu et al. ${ }^{1}$

cavity width closely. Integrating the effect of the transmission coefficient in the thermal conductance over the thermal factors in Eq. (7) will effectively perform this smoothing, so that the features will not be apparent in the thermal measurements. The sharp features are presumably also smoothed out if the junction is not perfectly abrupt. Results for $\mathcal{T}_{n}(\omega)$ for other values of $n$ are shown in Fig. 7. It is now straightforward to calculate the thermal conductivity using Eq. (7). We focus on the low-temperature limit where the universal result for $K / T$ applies in the ideal limit. With no reduction in the thermal transport due to scattering at the junction the plot of $K / T$ as a function of temperature develops a plateau at low temperatures at the universal value $\pi^{2} k_{B}^{2} / 3 h$ (see Fig. 8). In this regime the conductivity is dominated by the acoustic mode with $\omega \rightarrow 0$ as $k \rightarrow 0$. Scattering at the junction reduces the transmission of the small $\omega, k$ waves, so that the value of $K / T$ is reduced from the no-scattering value. As can be seen from Fig. 8 this reduction begins to occur as the temperature is lowered at about the same temperature at which the plateau in the ideal case begins to develop. This suggests that the plateau at the universal value of $K / T$ will not be well developed for the abrupt junction. The full calculation using Eq. (7) and the reduced transmission coefficients of all the modes (solid curve in Fig. 8) shows that this is the caseincluding the effects of scattering at the abrupt junction yields a $K / T$ curve that tends smoothly to zero as $T \rightarrow 0$. This result clearly demonstrates the importance of using smooth junctions between the bridge and the reservoirs, such as was done in the experiments of Schwab et al., ${ }^{5}$ if the universal value of $K / T$ is to be apparent.

\section{Long-Wavelength Calculation}

Although we have performed the full calculation of the transmission coefficient for the scalar case, this will not be 
possible for the elasticity-theory calculation. We therefore introduce a simple analytic technique for establishing the low-frequency limit of $\mathcal{T}_{0}(\omega)$ that can be extended to the full elasticity description.

The approach relies on the poor transmission $\mathcal{T}_{0}(\omega) \rightarrow 0$ for $\omega \rightarrow 0$ (long-wavelength waves are strongly affected by the abrupt junction) to treat the transmission perturbatively. First the bridge mode is calculated assuming perfect reflection, i.e., isolated from the supports. A simple analysis shows that the appropriate boundary condition on the end of the bridge is zero displacement (rather than zero stress). If we now "reconnect" the bridge to the supports, the stress fields at the end face of the bridge act as radiation sources of waves into the cavity. The total power in these waves for unit incident amplitude in the bridge gives us the transmission coefficient. The total power radiated may be readily calculated by integrating the product of the stress source and resulting velocity across the end face of the bridge.

To establish the appropriate boundary conditions in the $\mathcal{T}_{0}(\omega) \rightarrow 0$ limit we first consider a finite cavity width, i.e., an abrupt junction between a bridge of width $b$ for $x<0$ and a cavity of finite width $B \gg b$ for $x>0$ in the limit $\omega, k \rightarrow 0$. (It is simplest here to consider the transmission from bridge to cavity, and we have reversed the sense of the $x$ coordinate compared to Sec. II B 1.) For $x<0$ we consider an incident wave of unit amplitude and a reflected wave of amplitude $r$

$$
\phi=e^{i k x-i \omega t}+r e^{-i k x-i \omega t}, \quad x<0 .
$$

For $x>0$ there is only the transmitted wave

$$
\phi=t e^{i k x-i \omega t}, \quad x>0 .
$$

Here the wave numbers $k$ are fixed by the dispersion relation $\omega=c k$, which is the same on both sides of the junction for the acoustic mode.

The reflection and transmission amplitudes can be calculated by a simple matching at $x=0$. (This is equivalent to a wave impedance calculation.) Matching the displacement field $\phi$ gives

$$
1+r=t
$$

and matching the total force gives

$$
b(1-r)=B t .
$$

Note that the force (the integrated stress) is conserved because there are no additional stresses on the cavity face for $|y|>b / 2$ : this matching would not be appropriate for the boundary condition used by Angelescu et al. ${ }^{1}$

Thus we find

$$
r=-\frac{B-b}{B+b}, \quad t=\frac{2 b}{b+B} .
$$

These expressions are good for $k B, k b \ll 1$. In this limit the matching conditions can be applied outside of the region close to the junction where the fields are perturbed from their asymptotic forms, Eqs. (47) and (48), but before the exponential phase factors of the wave propagation have significantly changed. For $B \gg b$ these expressions reduce to $r \simeq$
$-1, t \simeq 0$, i.e., strong mismatch and almost perfect reflection with a sign change. Note that at $x=0$ this implies $\phi \simeq 0$, i.e., zero displacement boundary conditions, together with $\partial \phi / \partial x \simeq 2 i k$ for unit incident amplitude. We now use this result as the basis of the calculation of the transmission for $k$ small but nonzero and the limit $B \rightarrow \infty$.

The transmission at $k \neq 0$ is calculated as a radiation problem, namely, via the power radiated by the end of the bridge into the cavity. The zeroth-order approximation for the solution in the bridge is the perfect reflection result calculated above, giving the stress radiation source on the wall of the cavity

$$
s(y)= \begin{cases}\partial \phi / \partial x \simeq 2 i k e^{-i \omega t} & \text { for }|y|<b / 2, \\ 0 & \text { for }|y|>b / 2,\end{cases}
$$

where the second line is just the stress-free boundary condition

$$
\partial \phi / \partial x=0 \quad \text { for } x=0,|y|>b / 2 .
$$

The problem of the radiation due to a stress source on the boundary of an elastic half space is known as the Lamb problem in elasticity theory, and has been considered by many authors, for example, see Ref. 17. The radiation field can be calculated by standard Fourier-transform techniques. The solution in the cavity for $x>0$ can be written as

$$
\phi=e^{-i \omega t} \frac{1}{2 \pi} \int_{-\infty}^{\infty} \widetilde{\phi}(\zeta) e^{i q x} e^{i \zeta y} d \zeta,
$$

with

$$
q=\left\{\begin{array}{lll}
\sqrt{k^{2}-\zeta^{2}} & \text { for } & |\zeta| \leqslant k \\
i \sqrt{k^{2}-\zeta^{2}} & \text { for } & |\zeta|>k
\end{array}\right.
$$

corresponding to propagation or exponential decay away from the interface. The transform $\widetilde{\phi}(\zeta)$ is given by matching to the known $\partial \phi / \partial x$ at $x=0$ yielding

$$
i q \widetilde{\phi}(\zeta)=\int_{-\infty}^{\infty} s(y) e^{-i \zeta y} d y .
$$

The power radiated is the product of the stress $\partial \phi / \partial x$ and the velocity $\partial \phi / \partial t$ across the radiation source

$$
P=\left\langle\int_{-b / 2}^{b / 2} \frac{\partial \phi}{\partial t} \frac{\partial \phi}{\partial x} d y\right\rangle_{x=0},
$$

where the $\langle\cdots\rangle$ denotes the time average. For the fields varying as $e^{-i \omega t}$ this gives

$$
P=\left.\frac{1}{2} \operatorname{Re}\left[-i \omega \int_{-b / 2}^{b / 2} \phi(y) s^{*}(y) d y\right]\right|_{x=0} .
$$

Inserting the Fourier expression for $\phi(x=0)$,

$$
P=\frac{\omega}{4 \pi} \operatorname{Im} \int_{-\infty}^{\infty} d \zeta \widetilde{\phi}(\zeta) \int_{-\infty}^{\infty} d y s^{*}(y) e^{i \zeta y}
$$




$$
=\frac{\omega}{4 \pi} \operatorname{Im} \int_{-\infty}^{\infty} d \zeta \frac{1}{i q}\left|\int_{-\infty}^{\infty} s(y) e^{-i \zeta y} d y\right|^{2} .
$$

Since the transverse wave vector $\zeta$ is limited by $\zeta<k$ for the integrand to be imaginary (corresponding to $q$ real, i.e., propagating waves in the cavity), and $s(y)$ is nonzero only for $|y|<b / 2$, an expansion in $k b \ll 1$ is given by the expansion

$$
\int_{-\infty}^{\infty} s(y) e^{-i \zeta y} d y=s_{0}-i \zeta s_{1}+\cdots,
$$

where $s_{i}$ are successive moments of the source

$$
\begin{aligned}
& s_{0}=\int s(y) d y, \\
& s_{1}=\int s(y) y d y .
\end{aligned}
$$

We will need only the leading-order term, i.e., $s_{0}$ if the source is parity symmetric in $y, s_{1}$ if the source is antisymmetric. In particular for the present case $s_{0}=2 i k b$ so that

$$
P=\frac{\omega}{2 \pi} \int_{0}^{k} d \zeta \frac{4 k^{2}}{\sqrt{k^{2}-\zeta^{2}}} .
$$

Normalizing by the power in the incident wave $\frac{1}{2} b \omega k$ gives the transmission coefficient

$$
\mathcal{T}_{0}(\omega)=\frac{4 k b}{\pi} \int_{0}^{k} \frac{1}{\sqrt{k^{2}-\zeta^{2}}} d \zeta
$$

The integral expression for $\mathcal{T}_{0}$ can be easily understood physically as integrating over the power radiated into the continuum of waves, traveling at the wave propagation speed $c$, and propagating at all angles into the half space. For the scalar model there is a single type of propagating wave. With the full elasticity theory we will see a similar result, but with a number of propagating waves contributing to the power radiated.

Performing the integral gives

$$
\mathcal{T}_{0}(\omega)=2 k b=2 \omega b / c \quad \text { for } \quad k b \ll 1 .
$$

This result confirms the linear dependence on $\omega$ for small $\omega$, as shown in Fig. 7.

\section{THIN-PLATE THEORY}

A useful model of the mesoscopic geometry, that is more tractable than a fully three-dimensional elasticity calculation, is to assume a thin-plate geometry. Thus we take the elastic structure to be carved from a thin plate of uniform thickness $d$, which is taken to be small with respect to the other dimensions and also with respect to the wavelength of the elastic waves. In this model the mode frequency cutoffs at $k=0$, important in the thermal conductance, can be readily calculated (for most of the modes they are given by simple analytic expressions). In addition, although the mode structure is quite complicated, involving the mixing of longitudinal and transverse components by reflection at the edges, explicit expressions for the transverse structure of the modes in terms of a finite sum of sinusoidal functions can be written down. Compare this with a full three-dimensional analysis, where a finite-dimensional representation of the modes is not possible. In addition to the scattering at an abrupt junction pursued here, the thin-plate limit will permit an analysis of phenomena such as the scattering of the waves off surface and bulk imperfections, an issue that is pursued elsewhere. ${ }^{15}$

We first review the general elastic theory for waves in a thin plate, and confirm the expected dispersion relations for the acoustic modes of a rectangular beam (i.e., the "bridge") in the long-wavelength limit, and then use the equations to study the coupling of long-wavelength modes across the abrupt bridge-support junctions.

\section{A. Review of elastic theory and modes}

The elasticity of an isotropic solid is summarized by the relationship between the stress tensor $\mathbf{T}$ and the strain tensor

$$
T_{i j}=-K \Theta \delta_{i j}-2 \mu \Sigma_{i j}
$$

Here $\Theta$ is the dilation and $\boldsymbol{\Sigma}$ is the shear strain,

$$
\begin{gathered}
\Theta=\frac{\partial u_{x}}{\partial x}+\frac{\partial u_{y}}{\partial y}+\frac{\partial u_{z}}{\partial z}, \\
\Sigma_{i j}=\frac{1}{2}\left(\frac{\partial u_{i}}{\partial x_{j}}+\frac{\partial u_{j}}{\partial x_{i}}\right)-\frac{1}{3} \Theta \delta_{i j},
\end{gathered}
$$

with $\mathbf{u}(\mathbf{x})$ the displacement and $K$ and $\mu$ elastic constants.

For a thin plate of thickness $d$ in the $x y$ plane, linear elasticity theory can be separated into equations for the normal displacement $u_{z}=w(x, y)$ of the plate and for the inplane displacements averaged over the depth $\mathbf{u}(x, y)$ $=(u, v)$ with $u=\left\langle u_{x}(x, y, z)\right\rangle_{z}$ and $v=\left\langle u_{y}(x, y, z)\right\rangle_{z}$, all functions of just two spatial variables. This is done by assuming, for in-plane wave vectors $\mathbf{k}$ such that $k d \ll 1$, that the stresses in the vertical direction $\Sigma_{z j}$, which must be zero at the nearby stress-free top and bottom surfaces, may be put to zero everywhere. This allows the variation of the strains across the thickness of the plates to be eliminated in terms of the variables $u, v, w$. For example, setting $T_{z z}$ to zero gives

$$
\frac{\partial u_{z}}{\partial z}=-\frac{K-\frac{2}{3} \mu}{K+\frac{4}{3} \mu}\left(\frac{\partial u}{\partial x}+\frac{\partial v}{\partial y}\right)
$$

Thus the modes separate into modes with in-plane polarizations, and modes with polarizations normal to the plane (flexural modes). The full development can be found in any standard text on elasticity, for example Landau and Lifshitz ${ }^{16}$ or Graff. ${ }^{18}$ Here we collect the main results. 


\section{In-plane polarization}

Using relationships such as Eq. (69) leads to an effective two-dimensional elasticity theory for displacements in the plane, summarized by the stress-strain relationship

$$
T_{i j}^{(2)}=-\bar{K} \Theta^{(2)} \delta_{i j}-2 \bar{\mu} \Sigma_{i j}^{(2)},
$$

where the indices $i, j$ now run only over $x$ and $y, \Theta^{(2)}$ and $\Sigma^{(2)}$ are the two-dimensional dilation and shear strain tensor

$$
\begin{gathered}
\Theta^{(2)}=\frac{\partial u_{x}}{\partial x}+\frac{\partial u_{y}}{\partial y} \\
\Sigma_{i j}^{(2)}=\frac{1}{2}\left(\frac{\partial u_{i}}{\partial x_{j}}+\frac{\partial u_{j}}{\partial x_{i}}\right)-\frac{1}{2} \Theta^{(2)} \delta_{i j}
\end{gathered}
$$

and the effective two-dimensional elastic constants are

$$
\begin{gathered}
\bar{\mu}=\mu, \\
\bar{K}=\frac{3 K \mu}{K+\frac{4}{3} \mu} .
\end{gathered}
$$

The elastic waves with in-plane polarization are then given by the equation of motion

$$
\begin{aligned}
\rho \frac{\partial^{2} \mathbf{u}}{\partial t^{2}} & =-\nabla_{\perp} \cdot \mathbf{T}^{(2)} \\
& =\bar{K} \nabla_{\perp}\left(\nabla_{\perp} \cdot \mathbf{u}\right)+\bar{\mu} \nabla_{\perp}^{2} \mathbf{u},
\end{aligned}
$$

with $\nabla_{\perp}$ the horizontal gradient operator. In a horizontally infinite sheet there are longitudinal and transverse waves with speeds

$$
\begin{gathered}
c_{L}^{2}=\frac{\bar{K}+\bar{\mu}}{\rho}=\frac{4 \mu}{\rho} \frac{\left(K+\frac{1}{3} \mu\right)}{\left(K+\frac{4}{3} \mu\right)}, \\
c_{T}^{2}=\frac{\bar{\mu}}{\rho}=\frac{\mu}{\rho} .
\end{gathered}
$$

Alternatively, introducing Young's modulus $E$ and the Poisson ratio $\sigma$ (with $-1 \leqslant \sigma \leqslant \frac{1}{2}$ ) so that

$$
K=\frac{E}{3(1-2 \sigma)}, \quad \mu=\frac{E}{2(1+\sigma)},
$$

we have

$$
\begin{aligned}
c_{L}^{2} & =\frac{E}{\rho\left(1-\sigma^{2}\right)}, \\
c_{T}^{2} & =\frac{E}{2 \rho(1+\sigma)} .
\end{aligned}
$$

Rather than using the Poisson ratio directly it is convenient to introduce the parameter for the ratio of wave speeds

$$
r=\frac{c_{L}}{c_{T}}=\sqrt{\frac{2}{1-\sigma}}>1 .
$$

In terms of the component displacements $u, v$ and the parameter $r$ the elastic wave equation can be written

$$
\begin{aligned}
& \frac{1}{c_{T}^{2}} \frac{\partial^{2} u}{\partial t^{2}}=r^{2} \frac{\partial^{2} u}{\partial x^{2}}+\frac{\partial^{2} u}{\partial y^{2}}+\left(r^{2}-1\right) \frac{\partial^{2} v}{\partial x \partial y}, \\
& \frac{1}{c_{T}^{2}} \frac{\partial^{2} v}{\partial t^{2}}=\frac{\partial^{2} v}{\partial x^{2}}+r^{2} \frac{\partial^{2} v}{\partial y^{2}}+\left(r^{2}-1\right) \frac{\partial^{2} u}{\partial x \partial y} .
\end{aligned}
$$

A result that we will find useful later is for the component displacements in the solutions for the propagating waves

$$
\mathbf{u}= \begin{cases}u_{0}\left(1,-k_{T x} / k_{T y}\right) e^{i\left(\mathbf{k}_{T} \cdot \mathbf{x}-\omega t\right)} & \text { transverse wave } \\ u_{0}\left(1, k_{L y} / k_{L x}\right) e^{i\left(\mathbf{k}_{L} \cdot \mathbf{x}-\omega t\right)} & \text { longitudinal wave. }\end{cases}
$$

We note for completeness that in a three-dimensional elastic medium, waves polarized in the $x y$ plane having no $z$ dependence are also described by an effective twodimensional elasticity theory as in Eq. (70) but now with effective elastic constants $\bar{K}=K+\frac{1}{3} \mu, \bar{\mu}=\mu$ so that $c_{L}^{2} / c_{T}^{2}$ $=2(1-\sigma) /(1-2 \sigma)$. The difference in the effective elastic constants in the two cases arises because in the threedimensional medium restricted to no $z$ dependence, there can be no expansion in the $z$ direction to relieve the stresses set up by the strain in the $x y$ plane. In many elasticity textbooks, the "two-dimensional elasticity" and "thin-plate theory" discussed correspond to this case. We can relate these results to the thin-plate geometry we are considering by appropriately transforming the effective elastic constants.

For a finite plate we must apply stress-free boundary conditions at the side edges

$$
\hat{n} \cdot \mathbf{T}^{(2)}=0
$$

with $\hat{n}$ the normal to the edge. For waves propagating in the $x$ direction in a long finite plate of width $b$ the no-stress boundary condition at the edges of the plate at $y= \pm b / 2$ are therefore

$$
\begin{gathered}
T_{x y}^{(2)}=0=\bar{\mu}\left(\frac{\partial u}{\partial y}+\frac{\partial v}{\partial x}\right), \\
T_{y y}^{(2)}=0=\bar{\mu}\left(r^{2} \frac{\partial v}{\partial y}+\left(r^{2}-2\right) \frac{\partial u}{\partial x}\right) .
\end{gathered}
$$

The boundary conditions have the effect of reflecting incident longitudinal waves into both longitudinal and transverse reflected waves, so that these waves become coupled in the finite geometry, leading to a complicated dispersion relationship. The solutions propagating in the $x$ direction decouple into either an even or odd signature with respect to $y$ reflection, and take the form [using Eqs. (80)] 


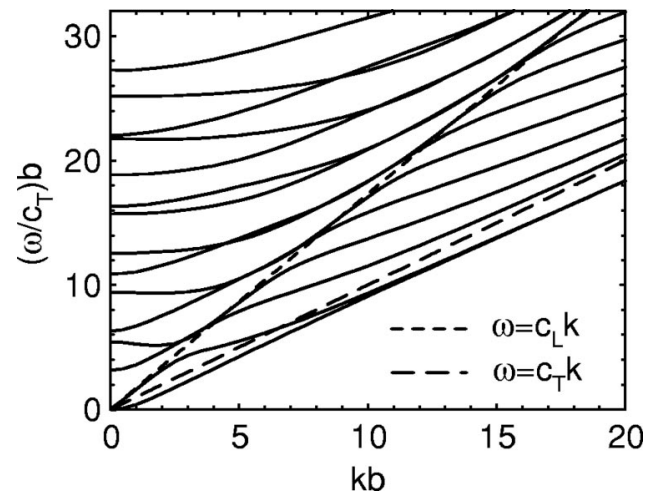

FIG. 9. Dispersion relation of in-plane polarized modes for the thin plate. The wave numbers are scaled by the bridge width $b$, and the frequencies by $b / c_{T}$ with $c_{T}$ the transverse wave speed in a large, thin plate. The dashed lines are the speeds of the transverse and longitudinal waves in a large thin plate. The value of the Poisson ratio is $\sigma=0.33$.

$$
\begin{aligned}
u^{(e)}= & {\left[a_{T}^{(e)} \cos \left(\chi_{T} y\right)+a_{L}^{(e)} \cos \left(\chi_{L} y\right)\right] e^{i(k x-\omega t)}, } \\
v^{(e)}= & {\left[\left(-i k / \chi_{T}\right) a_{T}^{(e)} \sin \left(\chi_{T} y\right)\right.} \\
& \left.+\left(i \chi_{L} / k\right) a_{L}^{(e)} \sin \left(\chi_{L} y\right)\right] e^{i(k x-\omega t)}
\end{aligned}
$$

and

$$
\begin{aligned}
u^{(o)}= & {\left[a_{T}^{(o)} \sin \left(\chi_{T} y\right)+a_{L}^{(o)} \sin \left(\chi_{L} y\right)\right] e^{i(k x-\omega t)}, } \\
v^{(o)}= & {\left[\left(i k / \chi_{T}\right) a_{T}^{(o)} \cos \left(\chi_{T} y\right)\right.} \\
& \left.-\left(i \chi_{L} / k\right) a_{L}^{(o)} \cos \left(\chi_{L} y\right)\right] e^{i(k x-\omega t)},
\end{aligned}
$$

where $\chi_{T, L}$ are given through the dispersion relation

$$
K^{2}=\omega^{2} / c_{T}^{2}=k^{2}+\chi_{T}^{2}=r^{2}\left(k^{2}+\chi_{L}^{2}\right)
$$

and we have defined $K=\omega / c_{T}$, the wave number of a transverse wave at the frequency $\omega$ in an infinite plate. The values of $\chi_{T, L}$ may be real or imaginary. Note that each wave combines both shear $\left(\chi_{T}\right)$ and compressional $\left(\chi_{L}\right)$ distortions, which are mixed by the reflection of the plane waves off the edges.

The amplitudes $a_{T, L}^{(e),(o)}$ must be adjusted to satisfy the boundary conditions at $y= \pm b / 2$, Eqs. (82). Substituting into these conditions leads to a system of two homogeneous equations for $a_{T}^{(e)}, a_{L}^{(e)}$ (and two for $a_{T}^{(o)}, a_{L}^{(o)}$ ) and so a consistency condition that leads to a transcendental equation for $\omega$ for each $k$, known as the Rayleigh-Lamb equations. ${ }^{20}$ For the even-signature modes the transcendental equation is

$$
\left(k^{2}-\chi_{T}^{2}\right)^{2} \tan \frac{\chi_{T} b}{2}+4 k^{2} \chi_{T} \chi_{L} \tan \frac{\chi_{L} b}{2}=0 .
$$

For the odd-signature modes the transcendental equation is

$$
4 k^{2} \chi_{T} \chi_{L} \tan \frac{\chi_{T} b}{2}+\left(k^{2}-\chi_{T}^{2}\right)^{2} \tan \frac{\chi_{L} b}{2}=0
$$

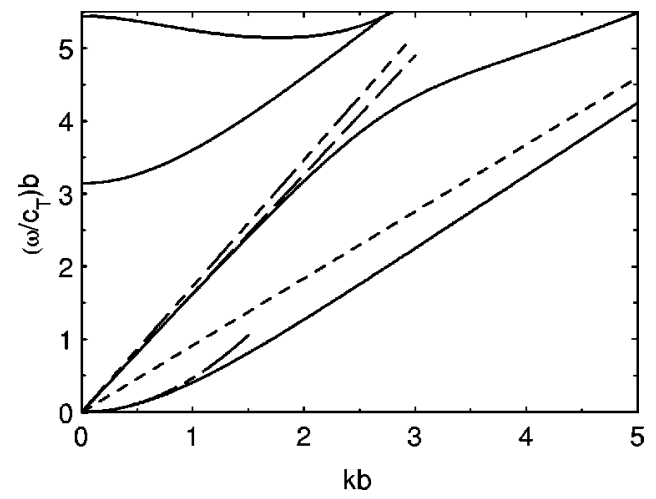

FIG. 10. Dispersion curves for the long-wavelength in-plane modes of a thin-plate beam of width $b$ for Poisson ratio $\frac{1}{3}$ (wave speed ratio $\sqrt{3}$ ). Solid lines: numerical results, long-dashed line: small- $k$ linear dispersion for compression mode, long-short-short dashed: linear dispersion for longitudinal wave in infinite plate, short dashed: linear dispersion for edge mode, and long-short dashed: quadratic dispersion for small- $k$ bending mode. Note the anomalous dispersion of the fourth mode at small $k$.

For a given value of the speed ratio $r$ these equations can be solved numerically for $\omega(k)$. The spectrum for the value of $r=\sqrt{3}$ corresponding to GaAs $\left(\sigma \simeq \frac{1}{3}\right)$ is shown in Figs. 9 and 10 .

At large values of $k$ the slopes of the curves, except for the lowest two, asymptote to $c_{L}$ or $c_{T}$ corresponding to the freely propagating waves in the plate. (This is not yet fully evident at the values of $\omega, k$ plotted in Fig. 9, but is confirmed by extending the numerics to higher values.) The lowest two modes on the other hand asymptote to a slope $c_{S}$ less than both $c_{T}$ and $c_{L}$. In this case for large $k$ we have $\tan \left(\chi_{T, L} b / 2\right) \rightarrow i$ in Eqs. (86) and (87) so that the slope $c_{S}$ $=r_{S} c_{T}$ is given by the solution of

$$
4 \sqrt{1-r_{S}^{2}} \sqrt{1-r_{S}^{2} / r^{2}}=\left(2-r_{S}^{2}\right)^{2}
$$

This is an edge wave analogous to the Rayleigh wave on the surface of a three-dimensional slab of material. For $r=\sqrt{3}$, Eq. (88) gives $c_{S}=\sqrt{2-2 / \sqrt{3}} c_{T}$.

The values of the finite-frequency intercepts of the "waveguide" modes for $k \rightarrow 0$ can also be calculated analytically. For the even mode intercepts, Eq. (86) is satisfied at $k \rightarrow 0$ by $\tan \left(\chi_{T} b / 2\right)=0$ or $\tan \left(\chi_{L} b / 2\right) \rightarrow \infty$. Similarly Eq. $(87)$ is satisfied by $\tan \left(\chi_{L} b / 2\right)=0$ or $\tan \left(\chi_{T} b / 2\right) \rightarrow \infty$. Thus the zero wave-number intercepts are given by the simple expressions for transverse and longitudinal wave propagation

$$
\omega_{n}^{(T)}=n \pi c_{T} / b, \quad \omega_{n}^{(L)}=r n \pi c_{T} / b=n \pi c_{L} / b .
$$

(These simple results hold because for $k=0$ there is no interconversion of longitudinal and transverse waves on reflection at the edges.) The shapes of the curves between $k=0$ and the large- $k$ asymptotes are quite complicated, with various mode crossings and regions of anomalous dispersion $d \omega / d k<0$.

We are particularly interested in the long-wavelength modes $k \rightarrow 0$. The dispersion relation in this limit can be 
found by Taylor expansion of the tan functions in Eqs. (86) and (87). The even mode tends to

$$
\omega \rightarrow 2 \sqrt{1-r^{-2}} c_{T} k=\sqrt{E / \rho} k .
$$

This agrees with the usual expression for the stretching mode of a rod. This is not the same as the dispersion for the bulk longitudinal modes in the thin plate, $\omega=c_{L} k$, but the speeds are quite close for $\sigma=0.33$. On the other hand the odd mode gives a quadratic dispersion, characteristic of bending modes of beams,

$$
\omega \rightarrow \sqrt{\frac{r^{2}-1}{3 r^{2}}} b c_{T} k^{2} .
$$

(This is given by expanding the tan functions up to cubic order.) Rod-bending theory gives the expression $\omega / k^{2}$ $=\sqrt{E I / \rho A}$ with $I$ the areal moment of inertia about the midline, and $A$ the cross-section area. For the rectangular beam we have $I / A=b^{2} / 12$, and using $\left(1-r^{-2}\right) c_{T}^{2}=E / 2 \rho$ shows the correspondence.

\section{Flexural modes}

The flexural modes are most easily derived by using relationships such as Eq. (69) to derive an expression for the energy of transverse displacements ${ }^{16}$

$$
\begin{aligned}
F= & \frac{1}{2} D \iint\left[\left(\nabla_{\perp}^{2} w\right)^{2}+2(1-\sigma)\left\{\left(\frac{\partial^{2} w}{\partial x \partial y}\right)^{2}\right.\right. \\
& \left.\left.-\frac{\partial^{2} w}{\partial x^{2}} \frac{\partial^{2} w}{\partial y^{2}}\right\}\right] d x d y,
\end{aligned}
$$

where

$$
D=\frac{E d^{3}}{12\left(1-\sigma^{2}\right)}
$$

is the flexural rigidity of the plate of thickness $d$. The equation of motion and boundary conditions are given by setting the variation of the energy with respect to displacements $w(x, y)$ to zero. For a region with rectangular boundaries at $x= \pm a / 2$ and $y= \pm b / 2$ the variation is

$$
\begin{aligned}
\delta F= & D \iint \nabla_{\perp}^{4} \omega \delta w d x d y-\int d y\left\{\delta w \left[\frac{\partial^{3} w}{\partial x^{3}}\right.\right. \\
& \left.\left.+(2-\sigma) \frac{\partial^{3} w}{\partial x \partial y^{2}}\right]-\frac{\partial \delta w}{\partial x}\left[\frac{\partial^{2} w}{\partial x^{2}}+\sigma \frac{\partial^{2} w}{\partial y^{2}}\right]\right\}\left.\right|_{x=-a / 2} ^{x=a / 2} \\
& -\int d x\left\{\delta w\left[\frac{\partial^{3} w}{\partial y^{3}}+(2-\sigma) \frac{\partial^{3} w}{\partial x^{2} \partial y}\right]\right. \\
& \left.-\frac{\partial \delta w}{\partial y}\left[\frac{\partial^{2} w}{\partial y^{2}}+\sigma \frac{\partial^{2} w}{\partial x^{2}}\right]\right\}\left.\right|_{y=-b / 2} ^{y=b / 2}
\end{aligned}
$$

The first term gives the effective force per unit area on the plate, and hence the equation of motion

$$
\rho d \frac{\partial^{2} w}{\partial t^{2}}+D \nabla_{\perp}^{4} w=0 .
$$

The last two terms on the right-hand side are boundary terms given by integration by parts. Physically they give the work done at the boundaries by the vertical force per length of boundary $V_{i}$ against the vertical displacement $\delta w$ and by the moment per unit length $M_{i}$ against the angular displacement of the plate ${ }^{21}-\vec{\nabla}_{i} w$. Thus at $x=a / 2$ we have

$$
\begin{gathered}
V_{x}=-D \frac{\partial}{\partial x}\left(\frac{\partial^{2} w}{\partial x^{2}}+(2-\sigma) \frac{\partial^{2} w}{\partial y^{2}}\right), \\
M_{x}=-D\left(\frac{\partial^{2} w}{\partial x^{2}}+\sigma \frac{\partial^{2} w}{\partial y^{2}}\right),
\end{gathered}
$$

and at $y=b / 2$

$$
\begin{gathered}
V_{y}=-D \frac{\partial}{\partial y}\left(\frac{\partial^{2} w}{\partial y^{2}}+(2-\sigma) \frac{\partial^{2} w}{\partial x^{2}}\right), \\
M_{y}=-D\left(\frac{\partial^{2} w}{\partial y^{2}}+\sigma \frac{\partial^{2} w}{\partial x^{2}}\right) .
\end{gathered}
$$

For free edges, these quantities must be set to zero. In addition to the force per unit length there are also point forces localized at the corners, e.g., at $x=a / 2, y= \pm b / 2$ (Ref. 19).

$$
F_{c}= \pm 2 D(1-\sigma) \frac{\partial^{2} w}{\partial x \partial y} .
$$

These must be included when we are calculating the total force acting across the width of the beam, for example,

$$
\begin{aligned}
F & =\int_{-b / 2}^{b / 2} V_{x} d y+F_{c}(b / 2)+F_{c}(-b / 2) \\
& =\frac{\partial}{\partial x} \int_{-b / 2}^{b / 2} M_{x} d y
\end{aligned}
$$

and the latter equality shows the consistency with the macroscopic equation for the rotational equilibrium of the beam (see Sec. III B 2 below).

We now calculate the modes propagating in the $x$ direction $w^{(e)}, w^{(o)} \propto e^{i(k x-\omega t)}$ in the bridge of width $b$. Again the modes have either even or odd signature with respect to $y$ reflections. Since the wave equation is fourth order in the spatial derivatives, for each frequency $\omega$ there are two even or odd components. The solutions to the wave equation are (even)

$$
w^{(e)}=\left[a_{+}^{(e)} \cosh \left(\chi_{+} y\right)+a_{-}^{(e)} \cosh \left(\chi_{-} y\right)\right] e^{i(k x-\omega t)}
$$

and (odd) 


$$
w^{(o)}=\left[a_{+}^{(o)} \sinh \left(\chi_{+} y\right)+a_{-}^{(o)} \sinh \left(\chi_{-} y\right)\right] e^{i(k x-\omega t)},
$$

where

$$
\chi_{ \pm}=\sqrt{k^{2} \pm K^{2}}
$$

and we have written

$$
\sqrt{\rho d / D} \omega=K^{2}
$$

Again the dispersion $\omega(k)$ and the ratio of amplitudes $a_{+} / a_{-}$are determined by the requirement of consistency with the boundary conditions at the edges $y= \pm b / 2$, Eq. (97). This gives for the even modes

$$
\begin{aligned}
{\left[K^{2}\right.} & \left.+(1-\sigma) k^{2}\right]^{2} \chi_{-} \tanh \left(\chi_{-} b / 2\right) \\
& =\left[K^{2}-(1-\sigma) k^{2}\right]^{2} \chi_{+} \tanh \left(\chi_{+} b / 2\right)
\end{aligned}
$$

and for the odd modes

$$
\begin{aligned}
{\left[K^{2}+\right.} & \left.(1-\sigma) k^{2}\right]^{2} \chi_{-} \operatorname{coth}\left(\chi_{-} b / 2\right) \\
& =\left[K^{2}-(1-\sigma) k^{2}\right]^{2} \chi_{+} \operatorname{coth}\left(\chi_{+} b / 2\right) .
\end{aligned}
$$

For $\omega \rightarrow 0$ we can expand the hyperbolic functions and solve algebraic equations to determine the dispersion curve.

For the even mode this gives $K^{2} / k^{2}=\sqrt{1-\sigma^{2}}$ yielding the quadratic dispersion of the beam bending mode

$$
\omega=\sqrt{\frac{D\left(1-\sigma^{2}\right)}{\rho d}} k^{2}=\sqrt{\frac{E}{12 \rho}} d k^{2},
$$

agreeing with the expression from simple rod theory. We can follow this mode to large $k$ where we find again a quadratic dispersion but with a different slope

$$
K^{2} / k^{2} \rightarrow \sqrt{\frac{1-3 \sigma+2 \sqrt{1-2 \sigma(1-\sigma)}}{(1-\sigma)^{2}(3+\sigma)}} .
$$

This is again an edge wave (now an edge bending wave). The intersections of the higher modes with the frequency axis are given by $k^{2} \rightarrow 0, \chi_{-} \rightarrow i K, \chi_{+} \rightarrow K$ so that Eq. (105) reduces to

$$
-\tan (K b / 2)=\tanh (K b / 2) .
$$

The solutions are well approximated by $K b=(3 / 2+2 n) \pi$ [i.e., $\omega=\sqrt{D / \rho d}(3 / 2+2 n)^{2} \pi^{2} / b^{2}$ ] for $n=0,1 \cdots$ (with an error of less than $\frac{1}{2} \%$ for the worst case $n=0$ ).

For the odd mode, Eq. (106) gives for $\omega, k \rightarrow 0$ the dispersion relation for the torsion mode

$$
\omega=\sqrt{\frac{D}{\rho d}} 2 \sqrt{6(1-\sigma)} \frac{k}{b}=2 \sqrt{\frac{\mu}{\rho}} \frac{d}{b} k,
$$

which agrees with the usual result calculated in elastic rod theory. The large- $k$ asymptote of this mode is the same as Eq. (108). The intersections of the higher odd modes with the frequency axis are from Eq. (106) given by

$$
\tan (K b / 2)=\tanh (K b / 2) \text {. }
$$

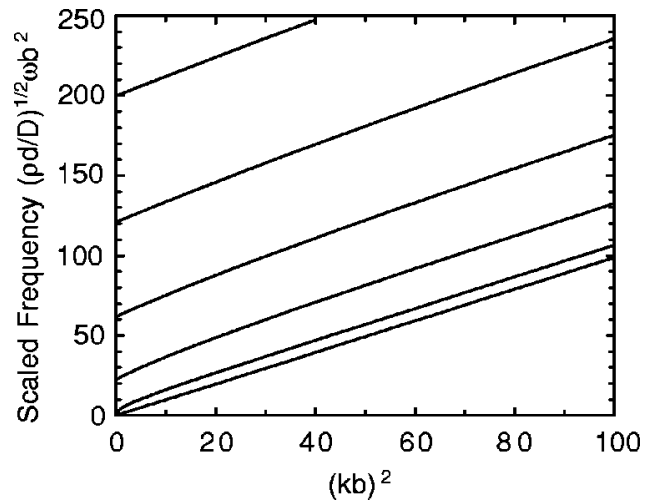

FIG. 11. Dispersion relation of the flexural modes of a thin plate with Poisson ratio $\sigma=0.33$. The wave numbers $k$ are scaled by the width $b$ and the frequencies by $(\rho d / D)^{1 / 2} b^{2}$. Note that the modes have approximately a quadratic wave-number dependence $\omega \simeq \omega_{c}^{2}$ $+\alpha k^{2}$ with $\alpha \simeq \sqrt{D / \rho d}$, corresponding to the "bulk" flexural wave. The two lower modes have a different form: one mode (the torsion mode) has a linear dispersion relation at low frequencies $\omega \propto k$ and both modes asymptote to $\omega=\beta k^{2}$ at large $k$ with $\beta<\alpha$ corresponding to an edge wave. It turns out that $\alpha \simeq \beta$ for the particular value of $\sigma$ used here.

The solutions are well approximated by $K b=(5 / 2+2 n) \pi$ [i.e., $\omega=\sqrt{D / \rho d}(5 / 2+2 n)^{2} \pi^{2} / b^{2}$ ] for $n=0,1 \cdots$.

Combining the even and odd modes, the zero wavenumber frequency intercepts can be written

$$
\omega_{n} \simeq c_{T} \frac{1}{\sqrt{6(1-\sigma)}}\left(\frac{3}{2}+n\right)^{2} \pi^{2} d / b^{2} .
$$

The dispersion curves for $\sigma=\frac{1}{3}$ corresponding to GaAs are shown in Figs. 11 and 12.

\section{B. Transmission coefficient in the infinite-wavelength limit}

The transmission coefficients for the acoustic modes in the long-wavelength limit and for finite cavity width can be readily calculated by the wave matching methods as for the scalar waves. We investigate the transmission of very long-

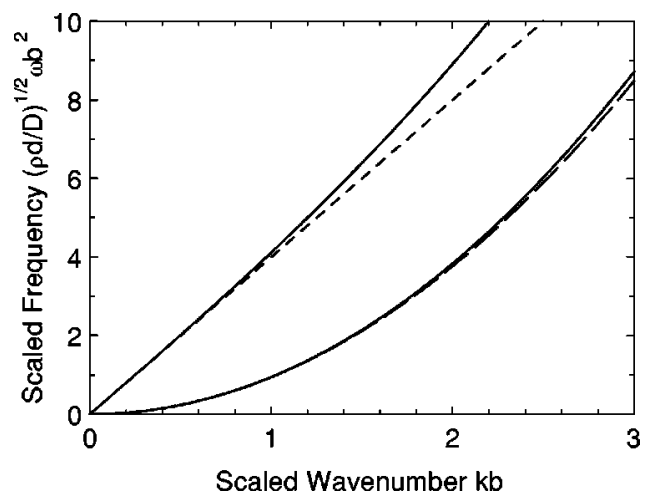

FIG. 12. Dispersion relation as in Fig. 11 but at small $\omega, k$. Note that the abscissa is proportional to $k$ in this plot. Solid lines: numerical results, long-dashed line: expected quadratic dispersion for rod-bending mode, and short-dashed line: expected linear dispersion for rod-torsion mode. 
wavelength modes at the abrupt junction between the bridge $(x<0)$ of width $b$ and the cavity $(x>0)$ of finite width $B$. It is easiest to evaluate the wave fields by simple macroscopic arguments. As well as providing the long-wavelength limit of the transmission coefficients, these results also provide the basis for calculating the leading-order finite $k$ corrections, following the same methods as in Sec. II B 2.

\section{Compression modes}

The compressional (extension) modes for $k b \rightarrow 0$ are given by the simple one dimensional calculation ${ }^{16}$

$$
\frac{\partial^{2} u}{\partial t^{2}}=c_{E}^{2} \frac{\partial^{2} u}{\partial x^{2}},
$$

where $c_{E}^{2}=E / \rho$.

For $x<0$ we have incident and reflected waves

$$
u=\left(e^{i k x}+r e^{-i k x}\right) e^{-i \omega t}
$$

and for $x>0$ a single transmitted wave

$$
u=t e^{i k x} e^{-i \omega t},
$$

where $\omega / k=c_{E}$. We match the displacement $u$ and the total force on either side of the interface

$$
\begin{gathered}
1+r=t, \\
i k b(1-r)=i k B t .
\end{gathered}
$$

Note that the force matching requires that the end surface of the cavity be stress free. This gives

$$
t=\frac{2}{1+h}, \quad r=-\frac{h-1}{h+1},
$$

where $h$ is the width ratio $h=B / b$. In the limit $h \rightarrow \infty$ we find $r \rightarrow-1, t \rightarrow 0$, i.e., perfect reflection with a sign change of the displacement. This implies that at the junction $x=0$ we have $u \simeq 0$ and the stress $E \partial u / \partial x \simeq 2 i k E e^{-i \omega t}$.

\section{Bending modes}

At long wavelengths both in-plane and flexural bending modes are given by equations for the total force $F$ and the total moment $M$ on each cross section. ${ }^{19}$ The moment from opposite forces on each end of a small element of the beam must cancel the net moment from the forces on the faces

$$
F=\frac{\partial M}{\partial x} .
$$

(Since the moments scale as the length of the element $\delta x$, whereas the moment of inertia scales as $\delta x^{2}$, there is no inertial term in this equation.) The net force on an element gives its acceleration

$$
\rho A \frac{\partial^{2} u}{\partial t^{2}}=\frac{\partial F}{\partial x},
$$

where $u$ is the bending displacement and $A$ is the crosssection area. The moment $M$ is given by the moment of the tensile stresses due to the extension and compression of the beam from its curvature

$$
M=-E I \frac{\partial^{2} u}{\partial x^{2}},
$$

where $I$ is the areal moment of inertia of the beam around the midline normal to the displacement. These equations together give the equation of motion

$$
\frac{\partial^{2} u}{\partial t^{2}}+\frac{E I}{\rho A} \frac{\partial^{4} u}{\partial x^{4}}=0 .
$$

The dispersion relation is quadratic, $\omega=\alpha k^{2}$ with $\alpha$ $=\sqrt{E I / \rho A}$. For a rectangular beam $I / A=d^{2} / 12$ with $d$ the thickness of the beam in the direction of the displacement. As we saw in the previous section, this reproduces the longwavelength limit of the acoustic bending modes calculated in thin-plate theory [Eq. (91) for the in-plane mode and Eq. (107) for the flexural mode].

At a frequency $\omega$, as well as the propagating modes at wave number $\pm \sqrt{\omega / \alpha}$, there are also evanescent modes with decay rate $\pm \sqrt{\omega / \alpha}$ : the modes localized at the junction and decaying to $\pm \infty$ must be included in the mode transmission problem.

For the bending mode with displacement normal to the plane the dispersion relation is the same in the bridge and cavity. Thus for an incident wave $e^{i(k x-\omega t)}$ in the bridge with $k=\sqrt{\omega / \alpha}$ we have

$$
u= \begin{cases}e^{i k x}+r e^{-i k x}+d_{-} e^{k x} & x<0, \\ t e^{i k x}+d_{+} e^{-k x} & x>0 .\end{cases}
$$

At the junction we require continuity of the displacement $u$, the rotation angle $\partial u / \partial x$, the total moment $-E I \partial^{2} u / \partial x^{2}$, and the total force $-E I \partial^{3} u / \partial x^{3}$. This gives the matrix equation

$$
\left[\begin{array}{cccc}
1 & -1 & 1 & -1 \\
1 & 1 & i & i \\
1 & -h & -1 & h \\
1 & h & -i & -i h
\end{array}\right]\left[\begin{array}{c}
r \\
t \\
d_{-} \\
d_{+}
\end{array}\right]=\left[\begin{array}{c}
-1 \\
1 \\
-1 \\
1
\end{array}\right]
$$

where $h=I_{c} / I_{b}=B / b$ is the ratio of the appropriate moment of inertia for the cavity and bridge. In the limit of large $h$ the solution is easily found to be

$$
\begin{gathered}
r=i, \\
t=4 / h, \\
d_{-}=-(1+i), \\
d_{+}=2(1-i) / h .
\end{gathered}
$$


For the bending mode with displacements in the plane of the plate the dispersion relation is different in bridge and cavity. For frequency $\omega$ the wave numbers in the bridge and cavity are $k_{b}, k_{c}$ with

$$
k_{c} / k_{b}=\left(\alpha_{b} / \alpha_{c}\right)^{1 / 2}=(b / B)^{1 / 2} .
$$

On the other hand the ratio of the moments of inertia is

$$
I_{c} / I_{b}=(B / b)^{3} .
$$

Thus the continuity of the displacement $u$, the rotation angle $\partial u / \partial x$, the total moment $-E I \partial^{2} u / \partial x^{2}$, and the total force $-E I \partial^{3} u / \partial x^{3}$ gives the matrix equation (writing $\bar{h}=\sqrt{B / b}$ )

$$
\left[\begin{array}{cccc}
1 & -1 & 1 & -1 \\
1 & \bar{h}^{-1} & i & i \bar{h}^{-1} \\
1 & -\bar{h}^{4} & -1 & \bar{h}^{4} \\
1 & \bar{h}^{3} & -i & -i \bar{h}^{3}
\end{array}\right]\left[\begin{array}{c}
r \\
t \\
d_{-} \\
d_{+}
\end{array}\right]=\left[\begin{array}{c}
-1 \\
1 \\
-1 \\
1
\end{array}\right] .
$$

In the limit of large $\bar{h}$ the solution is

$$
\begin{gathered}
r=i, \\
t=2 / \bar{h}^{3}, \\
d_{-}=-(1+i), \\
d_{+}=2 / \bar{h}^{3} .
\end{gathered}
$$

With these expressions in both cases we have at $x=0$ to leading order in $h^{-1}$ or $\bar{h}^{-1}$

$$
\begin{gathered}
u=0, \\
\partial u / \partial x=0, \\
\partial^{2} u / \partial x^{2}=-2(1+i) k^{2}, \\
\partial^{3} u / \partial x^{3}=-2(1+i) k^{3},
\end{gathered}
$$

so that the displacements $u$ and the angle $\partial u / \partial x$ tend to zero, but the corresponding stresses are large. Note that although the force and moment are out of phase for the single wave $e^{i(k x-\omega t)}$, because of the evanescent waves near the junction they are in phase at the junction plane $x=0$. Equations (130) become the zeroth-order input for the radiation calculation at nonzero wave number.

\section{Torsion modes}

The long-wavelength limit of torsion waves is described by the one-dimensional wave equation giving the angular acceleration in terms of the torque

$$
I \frac{\partial^{2} \theta}{\partial t^{2}}=C \frac{\partial^{2} \theta}{\partial x^{2}} .
$$

Here $C$ is the torsional rigidity giving the torque on a section due to the twist $\tau=\partial \theta / \partial x$

$$
\text { torque }=C \tau \text {. }
$$

It is given by ${ }^{16}$

$$
C=4 \mu \int \chi d x d y,
$$

where $\chi$ satisfies the equation in the cross section

$$
\nabla_{\perp}^{2} \chi=-1
$$

and $\chi=0$ on the boundaries. The form of the solution $\chi$ is the same as the profile of the flow of a viscous fluid through the section and $C$ is then proportional to the integrated flux. For the thin-plate geometry with thickness $d$ and width $b$ $\gg d$, the value of $C$ is $\frac{1}{3} \mu d^{3} b$ and value of $I$ is $\frac{1}{12} d b^{3}$, so that the ratio of propagation speeds in bridge and cavity is again the width ratio $h=B / b$.

It is interesting to evaluate the stress distribution for the thin plate. The stresses in the $(y, z)$ section are given in terms of $\chi$ by $^{16}$

$$
\begin{gathered}
\sigma_{y x}=2 \mu \tau \partial \chi / \partial z, \\
\sigma_{z x}=-2 \mu \tau \partial \chi / \partial y .
\end{gathered}
$$

The solution for $\chi$ is analogous to Poiseuille flow, so that

$$
\chi=\frac{1}{8}\left(d^{2}-4 z^{2}\right)
$$

except within a distance $\delta \sim d$ from the side wall, where $\chi$ must decrease to zero. Thus there is a distributed stress acting in the $y$ direction

$$
\sigma_{y x} \simeq-2 \mu \tau z
$$

and a stress in the $z$ direction that is effectively localized (within a distance $d$ ) at the edge

$$
\sigma_{z x} \simeq \frac{1}{4} \mu \tau\left(d^{2}-4 z^{2}\right) \delta(y-b / 2) .
$$

This localized stress corresponds to the corner forces, Eq. (98), in the thin-plate theory.

For an incident torsion wave $\theta=e^{i\left(k_{b} x-\omega t\right)}$ in the bridge for $x<0$ we have incident and reflected waves

$$
\theta=\left(e^{i k_{b} x}+r e^{-i k_{b} x}\right) e^{-i \omega t}
$$

and for $x>0$ a single transmitted wave

$$
\theta=t e^{i k_{c} x} e^{-i \omega t}
$$

where $k_{c} / k_{b}=B / b$. The matching of the angular displacement $\theta$ and torque $C \partial \theta / \partial x$ for an incident wave gives

$$
\begin{gathered}
1+r=t, \\
i k_{b} b(1-r)=i k_{c} B t
\end{gathered}
$$

so that

$$
t=\frac{2}{1+h^{2}}, \quad r=-\frac{h^{2}-1}{h^{2}+1},
$$


with $h=B / b$. In the limit $h \rightarrow \infty$ we find $r \rightarrow-1, t \rightarrow 0$, implying $\theta(x=0) \simeq 0$ and the stress $C \partial \theta / \partial x \simeq 2 i k C e^{-i \omega t}$.

\section{Transmission coefficient for small $b$}

In this section we calculate the small wave-vector asymptotic limit of the transmission coefficient from the four acoustic modes of the beam into the cavity. The method follows that of Sec. II B 2. Thus we calculate the radiation from oscillating stresses $s(y) e^{-i \omega t}$ on the edge of the cavity. The stresses are calculated as the stresses arising on the ends of the bridge for zero displacement boundary conditions, as follows from the analyses in the previous section of the modes at infinite wavelengths coupling into a cavity of finite width. For the long-wavelength value of the transmission coefficients, only the radiation by the integrated stress $\int s(y) d y$ for the even-parity modes, or the integrated first moment $\int s(y) y d y$ for the odd-parity modes, is needed. The Lamb problem of the radiation from surface sources into an elastic half space has been much studied in the literature, for example, see (Ref. 17) and the reader is referred there for a more exhaustive discussion of this aspect of the calculation. The details of the calculations are quite complicated, and the reader may choose to skip these sections and refer to the discussion of the results in Sec. IV and the summary in Table I there.

\section{In-plane compression}

For a compressional wave in the bridge of unit incident amplitude in the displacement, the oscillating end of the bridge acts as a stress source on the cavity face of amplitude $2 i E k$ over the source region $|y|<b / 2$, embedded in the otherwise stress-free line $x=0$. The solutions to the wave equations (79) in the cavity can be written [cf. Eq. (80)]

$$
u=\frac{1}{2 \pi} \int_{-\infty}^{\infty}\left[a_{T}(\zeta) e^{i k_{T} x}+a_{L}(\zeta) e^{i k_{L} x}\right] e^{i \zeta y} e^{-i \omega t} d \zeta
$$

$$
\begin{aligned}
v= & \frac{1}{2 \pi} \int_{-\infty}^{\infty}\left[-\left(k_{T} / \zeta\right) a_{T}(\zeta) e^{i k_{T^{x}}}\right. \\
& \left.+\left(\zeta / k_{L}\right) a_{L}(\zeta) e^{i k_{L} x}\right] e^{i \zeta y} e^{-i \omega t} d \zeta,
\end{aligned}
$$

where $k_{T}$ and $k_{L}$ are the $x$ components of the wave vectors of the transverse and longitudinal components

$$
k_{T}=\left\{\begin{array}{ll}
\sqrt{K^{2}-\zeta^{2}} & |\zeta| \leqslant K, \\
i \sqrt{\zeta^{2}-K^{2}} & |\zeta|>K,
\end{array} k_{L}=\left\{\begin{array}{lr}
\sqrt{K^{2} / r^{2}-\zeta^{2}} & |\zeta| \leqslant K / r, \\
i \sqrt{\zeta^{2}-K^{2} / r^{2}} & |\zeta|>K / r
\end{array}\right.\right.
$$

with $K^{2}=\omega^{2} / c_{T}^{2}$. (The signs chosen correspond to waves propagating away or exponentially decaying.) The amplitudes $a_{L, T}$ are fixed by matching to the normal and tangential stress sources $\Sigma_{n}=-T_{x x}^{(2)}$ and $\Sigma_{t}=-T_{y x}{ }^{(2)}$ for $|y|<b / 2$

$$
\begin{gathered}
\bar{\mu}\left(r^{2} \frac{\partial u}{\partial x}+\left(r^{2}-2\right) \frac{\partial v}{\partial y}\right)=\Sigma_{n}, \\
\bar{\mu}\left(\frac{\partial u}{\partial y}+\frac{\partial v}{\partial x}\right)=\Sigma_{t} .
\end{gathered}
$$

In the present case $\Sigma_{n}=2 i E k e^{-i \omega t}$ for unit incident wave amplitude and $\Sigma_{t}=0$. Both components of the stress are zero for $|y|>b / 2$. Taking the leading-order expansion in $K b$ for the Fourier transform of the source stress as in the scalar calculation, Sec. II B 2, gives for the Fourier components

$$
\begin{array}{r}
r^{2}\left(i k_{T} a_{T}+i k_{L} a_{L}\right)+\left(r^{2}-2\right)\left(-i k_{T} a_{T}+i \zeta^{2} a_{L} / k_{L}\right)=\Sigma_{n} b / \mu, \\
i \zeta\left(a_{T}+a_{L}\right)+\left(-i k_{T}^{2} a_{T} / \zeta+i \zeta a_{L}\right)=0 .
\end{array}
$$

These equations are readily solved for $a_{L, T}$ from which we can calculate $u(x=0)$ for unit driving stress

$$
\frac{u(x=0)}{\Sigma_{n}}=-\frac{r^{2} b}{\bar{\mu}} \frac{i}{\pi} \int_{0}^{\infty} \frac{\kappa_{L}(\xi)}{F_{o}(\xi)} d \xi,
$$

where $\xi=r \zeta / K$ is the $y$-wave vector scaled by the longitudinal wave number $K / r=\omega / c_{L}$,

$$
F_{0}(\xi)=\left(2 \xi^{2}-r^{2}\right)^{2}+4 \xi^{2} \kappa_{T}(\xi) \kappa_{L}(\xi)
$$

and

$$
\kappa_{T}=\left\{\begin{array}{ll}
\sqrt{r^{2}-\xi^{2}} & |\xi| \leqslant r, \\
i \sqrt{\xi^{2}-r^{2}} & |\xi|>r,
\end{array} \kappa_{L}= \begin{cases}\sqrt{1-\xi^{2}} & |\xi| \leqslant 1, \\
i \sqrt{\xi^{2}-1} & |\xi|>1 .\end{cases}\right.
$$

[This result is analogous to Eq. (123) of Miller and Pursey ${ }^{17}$ who calculate the average displacement at the aperture per unit oscillating stress for a line on a threedimensional half space. Indeed we can use their result if we express it in terms of the ratio of wave speeds, and the elastic constant $\mu$ which retains its significance unchanged between the two geometries. The translation from their (MP) notation is then $u_{x, M P} \rightarrow\left(\omega / c_{L}\right) u_{x}, \quad a_{M P} \rightarrow\left(\omega / c_{L}\right) b / 2, \quad \mu_{M P} \rightarrow r$, $c_{44, M P} \rightarrow \bar{\mu}$. Note carefully that the usage of " $\mu$ " is different in their work and ours. We have also taken the leading-order term in $\omega b / c_{L}$ by making the replacement $e^{i \xi y} \rightarrow 1$ for $|y|$ $<b / 2$.]

For unit incident wave in the beam the longitudinal stress at the aperture is $2 i E k e^{-i \omega t}$ and the power radiated is $\frac{1}{2} b \operatorname{Re}\left(-\dot{u} \Sigma_{n}^{*}\right)$. In the incident wave the stress is $i E k e^{-i \omega t}$ and the velocity $\dot{u}_{x}$ is $-i \omega e^{-i \omega t}$ so that the incident power is $\frac{1}{2} b E \omega k$. The transmission coefficient is therefore

$$
\mathcal{T}_{0}(\omega)=4(k b)\left[\frac{4}{\pi} \frac{(1+\sigma)}{(1-\sigma)} \operatorname{Re} \int_{0}^{\infty} \frac{\kappa_{L}(\xi)}{F_{0}(\xi)} d \xi\right],
$$

where we have used $E / \bar{\mu}=2(1+\sigma)$ and $r^{2}=2 /(1-\sigma)$. The various contributions to the the integral are easily understood in terms of the different waves radiated into the cavity. Remember that $\xi$ is the $y$-wave number of the radiated waves in units of $k_{L}$. There are contributions to the integral for 0 $<\xi<r$ corresponding to the radiation of transverse and longitudinal waves over all angles. In addition there is a contribution from the residue of the pole at $F_{0}(\xi)=0$ which cor- 
responds to the radiation of edge waves. Miraculously, the quantity in the square brackets numerically evaluates to 1.0 independent of $\sigma$ in the allowed range $-1<\sigma<\frac{1}{2}$, so that

$$
\mathcal{T}_{0}(\omega)=4 k b
$$

\section{In-plane bending}

For an incident bending wave with unit displacement amplitude $v$ in the $y$ direction there are two sources of radiation into the cavity: the oscillating moment $2 \sqrt{2} E I k^{2} e^{-i \omega t}$ and the oscillating shear (tangential) force $2 \sqrt{2} E I k^{3} e^{-i \omega t}$ over the source width $b$ in the cavity wall. The moment can be described in terms of the normal stress $2 \sqrt{2} E k^{2} e^{-i \omega t} y$ since $\int y^{2} d y=I$. The tangential force has an additional power of $k \sim \sqrt{\omega}$ compared to this, but the radiation efficiency of the normal force is reduced by a power of $k_{c} b$ with $k_{c}$ the wave number of a propagating mode in the cavity $k_{c} \sim \omega / c_{T}$, since the two halves of the radiation source cancel at leading order. This means that the contribution of the normal stress to the power radiated is higher order in $\omega$ for small $\omega$, and may be neglected.

The analysis proceeds as in the previous section. We again use Eqs. (145) but now with $\Sigma_{t}=2 \sqrt{2} E(I / b) k^{3} e^{-i \omega t}$ and $\Sigma_{n} \simeq 0$. This gives the equations for the mode amplitudes

$$
\begin{gathered}
r^{2}\left(i k_{T} a_{T}+i k_{L} a_{L}\right)+\left(r^{2}-2\right)\left(-i k_{T} a_{T}+i \zeta^{2} a_{L} / k_{L}\right)=0, \\
i \zeta\left(a_{T}+a_{L}\right)+\left(-i k_{T}^{2} a_{T} / \zeta+i \zeta a_{L}\right)=\Sigma_{t} b / \bar{\mu},
\end{gathered}
$$

which can be solved to yield the response to leading order

$$
\frac{v(x=0)}{\Sigma_{t}} \simeq-\frac{r^{2} b}{\bar{\mu}} \frac{i}{\pi} \int_{0}^{\infty} \frac{\kappa_{T}(\xi)}{F_{o}(\xi)} d \xi
$$

[This result is analogous to Eq. (124) of Miller and Pursey. $\left.{ }^{17}\right]$ This gives the average power radiated to leading order in small $\omega$

$$
P_{r a d}=\frac{1}{2} b \operatorname{Re}\left(-\dot{v} \Sigma_{t}^{*}\right)=\frac{1}{2} b\left(2 \sqrt{2} E I k^{3} / b\right)^{2} \operatorname{Re}\left(i \omega v / \Sigma_{t}\right) .
$$

For the incident wave of unit amplitude we have

$$
\left(u, u^{\prime}, M, F\right)=\left(1, i k, E I k^{2}, i E I k^{3}\right) e^{i(k x-\omega t)}
$$

so that the average incident power is

$$
P_{i n c}=\frac{1}{2} \operatorname{Re}\left[-i \omega\left(F u^{*}+M u^{\prime} *\right)\right]=\omega E I k^{3} .
$$

The ratio gives the transmission coefficient

$$
\mathcal{T}_{o}(\omega)=\frac{1}{3}(b k)^{3}\left[\frac{4}{\pi} \frac{(1+\sigma)}{(1-\sigma)} \operatorname{Re} \int_{0}^{\infty} \frac{\kappa_{T}(\xi)}{F_{0}(\xi)} d \xi\right] .
$$

Again the quantity in the braces turns out to be unity, so we have

$$
\mathcal{T}_{o}(\omega)=\frac{1}{3}(b k)^{3}
$$

\section{Flexural modes}

The flexural displacement $w(x, y)$ for a wave at frequency $\omega$ satisfies the equation

$$
\nabla^{4} w=K^{4} w
$$

with $K^{2}=\sqrt{\rho d / D} \omega$, cf. Sec. III A 2. Expanding the cavity solution in transverse Fourier modes

$$
w(x, y)=\frac{1}{2 \pi} \int_{-\infty}^{\infty} \tilde{w}(x, \zeta) e^{i \zeta y} d \zeta
$$

the Fourier amplitude $\tilde{w}$ satisfies

$$
\left(\partial^{2} / \partial x^{2}-\zeta^{2}\right)^{2} \tilde{w}=K^{4} \tilde{w} .
$$

The solutions are $\tilde{w} \sim e^{i k x}$ with

$$
k^{2}= \pm K^{2}-\zeta^{2}
$$

Solutions corresponding to a wave propagating away from the source at $x=0$ or exponentially decaying to $+\infty$ are given by $k_{ \pm}$as

$$
k_{+}=i \sqrt{K^{2}+\zeta^{2}}
$$

and

$$
k_{-}= \begin{cases}\sqrt{K^{2}-\zeta^{2}} & \zeta^{2}<K^{2}, \\ i \sqrt{\zeta^{2}-K^{2}} & \zeta^{2}>K^{2}\end{cases}
$$

Thus the solution in the cavity can be written

$$
w(x, y)=\frac{1}{2 \pi} \int_{-\infty}^{\infty}\left[\tilde{w}_{+}(\zeta) e^{i k_{+} x}+\tilde{w}_{-}(\zeta) e^{i k_{-} x}\right] e^{i \zeta y} d \zeta .
$$

The sources at $x=0$ are a moment $M(y)$ and an effective force per unit length $V(y)$,

$$
\begin{gathered}
M(y)=-D\left(\frac{\partial^{2} w}{\partial x^{2}}+\sigma \frac{\partial^{2} w}{\partial y^{2}}\right), \\
V(y)=-D\left(\frac{\partial^{3} w}{\partial x^{3}}+(2-\sigma) \frac{\partial^{3} w}{\partial x \partial y^{2}}\right) \\
+b^{-1}\left[F_{c}(b / 2)+F_{c}(-b / 2)\right],
\end{gathered}
$$

with Fourier transforms $\widetilde{M}(\zeta)$ and $\widetilde{V}(\zeta)$. The last two terms in the second equation are the corner forces. Matching these boundary conditions gives 


$$
\begin{gathered}
\quad\left[K^{2}+\zeta^{2}(1-\sigma)\right] \tilde{w}_{+}(\zeta)-\left[K^{2}-\zeta^{2}(1-\sigma)\right] \tilde{w}_{-}(\zeta) \\
=-\tilde{M}(\zeta) / D, \\
i k_{+}\left[K^{2}-\zeta^{2}(1-\sigma)\right] \tilde{w}_{+}(\zeta)-i k_{-}\left[K^{2}+\zeta^{2}(1-\sigma)\right] \tilde{w}_{-}(\zeta) \\
=-\widetilde{V}(\zeta) / D .
\end{gathered}
$$

The average power radiated is

$$
P=-\left\langle\int \dot{w}(x=0, y) V(y)+\dot{\theta}(x=0, y) M(y) d y\right\rangle_{t},
$$

with $\theta=-\partial w / \partial x$ the tilt angle and the dot denoting a time derivative. For oscillations $e^{-i \omega t}$ the average over time gives

$$
\begin{aligned}
P= & P_{w}+P_{\theta} \\
= & -\frac{1}{2} \omega \operatorname{Im}\left[\int w(x=0, y) V^{*}(y) d y\right. \\
& \left.+\int \theta(x=0, y) M^{*}(y) d y\right] .
\end{aligned}
$$

Evaluating the first integral in terms of Fourier expansions we find the power radiated by the force

$$
P_{w}=-\frac{1}{4 \pi} \omega \operatorname{Im}\left[\int_{-\infty}^{\infty} d \zeta\left[\tilde{w}_{+}(\zeta)+\tilde{w}_{-}(\zeta)\right] \widetilde{V}^{*}(\zeta)\right]
$$

As in the scalar case, the imaginary part of this integral corresponds to the excitation of propagating waves for which $\zeta<K$ and we may evaluate $\widetilde{V}(\zeta)$ to lowest nonzero order in $K b$

$$
\widetilde{V}(\zeta)=\int_{-b / 2}^{b / 2} d y V(y) e^{-i \zeta y} \simeq V_{0}-i \zeta V_{1}+\cdots
$$

with

$$
\begin{gathered}
V_{0}=\int d y V(y), \\
V_{1}=\int d y y V(y) .
\end{gathered}
$$

We only keep the second term for antisymmetric sources for which $V_{0}$ is zero. Note that $V_{0}$ is the total force normal to the plate, and $V_{1}$ is the torque about the $x$ axis, and these can be evaluated from macroscopic arguments.

Similar arguments for $P_{\theta}$ give

$$
P_{\theta}=\frac{1}{4 \pi} \omega \operatorname{Im}\left[\int_{-\infty}^{\infty} d \zeta\left[i k_{+} \tilde{w}_{+}(\zeta)+i k_{-} \tilde{w}_{-}(\zeta)\right] \tilde{M}^{*}(\zeta)\right],
$$

with

$$
\tilde{M}(\zeta) \simeq M_{0}-i \zeta M_{1}+\cdots,
$$

with $M_{0}, M_{1}$ the zeroth and first moments of $M$ over the bridge end. Now we can calculate explicit results for the bending and torsion modes.

a. Bending mode. From Eq. (130) we see that an incident wave of unit displacement amplitude $e^{i(k x-\omega t)}$ in the bridge gives oscillating sources on the edge of the cavity

$$
\begin{aligned}
& M_{0}=2 \sqrt{2} D\left(1-\sigma^{2}\right) b k^{2} e^{i \pi / 4} e^{-i \omega t}, \\
& V_{0}=2 \sqrt{2} D\left(1-\sigma^{2}\right) b k^{3} e^{i \pi / 4} e^{-i \omega t}
\end{aligned}
$$

Note that we are using the macroscopic formulation to derive these expressions. It is somewhat subtle to directly use the expressions, Eq. (166), since the $y$ dependence of the mode structure cannot be ignored. We have verified that these expressions are reproduced using the long-wavelength limit of the mode structure given by solving Eqs. (101) and (102). Now defining $\xi=\zeta / K$ and

$$
\tilde{w}_{ \pm}(\zeta)=-2 \sqrt{2} b e^{i \pi / 4} u_{ \pm}(\xi),
$$

using the dispersion relation $k^{2}=K^{2} / \sqrt{1-\sigma^{2}}$, and then matching to the sources gives

$$
\begin{aligned}
& {\left[1+\xi^{2}(1-\sigma)\right] u_{+}-\left[1-\xi^{2}(1-\sigma)\right] u_{-}=\left(1-\sigma^{2}\right)^{1 / 2}} \\
& -\sqrt{1+\xi^{2}}\left[1-\xi^{2}(1-\sigma)\right] u_{+}-i \sqrt{1-\xi^{2}}\left[1+\xi^{2}(1-\sigma)\right] u_{-} \\
& =\left(1-\sigma^{2}\right)^{1 / 4} .
\end{aligned}
$$

The power radiated is

$$
\begin{aligned}
P= & \omega D b^{2} k^{2} K^{2}\left(1-\sigma^{2}\right) \frac{2}{\pi} \operatorname{Im}\left[\int_{-\infty}^{\infty}\left(u_{+}+u_{-}\right)\left(1-\sigma^{2}\right)^{-1 / 4}\right. \\
& \left.-\left(-\sqrt{1+\xi^{2}} u_{+}+i \sqrt{1-\xi^{2}} u_{-}\right) d \xi\right]
\end{aligned}
$$

Normalizing by the incident power $P_{i}=\omega D b k^{3}\left(1-\sigma^{2}\right)$ gives the transmission coefficient

$$
T_{0}(\omega)=k b I_{1}(\sigma),
$$

where $I_{1}$ is the integral

$$
\begin{aligned}
I_{1}(\sigma)= & \sqrt{1-\sigma^{2}} \frac{2}{\pi} \operatorname{Im} \int_{-\infty}^{\infty}\left[\left(u_{+}+u_{-}\right)\left(1-\sigma^{2}\right)^{-1 / 4}\right. \\
& \left.+\left(\sqrt{1+\xi^{2}} u_{+}-i \sqrt{1-\xi^{2}} u_{-}\right)\right] d \xi
\end{aligned}
$$

For $\sigma=0.33$, evaluating $u_{ \pm}$from Eq. (179) we find

$$
I_{1}=2.3 \quad(\sigma=0.33) .
$$

b. Torsion mode. A unit amplitude mode $\theta=e^{i(k x-\omega t)}$ gives the oscillating torque source

$$
\tau=4 D b(1-\sigma) i k e^{-i \omega t} .
$$

Here the wave number in the beam is given by [cf. Eq. (110)]

$$
k b=\frac{1}{2 \sqrt{6(1-\sigma)}}(K b)^{2},
$$


TABLE I. Long-wavelength properties of the modes of a thin beam. See text for the details.

\begin{tabular}{|c|c|c|c|c|}
\hline Mode & $\omega(k \rightarrow 0) / c_{T} k$ & $\omega_{1} /\left(\pi c_{T} / b\right)$ & $\mathcal{T}_{o}(k \rightarrow 0)$ & $\mathcal{T}_{o}(\omega \rightarrow 0), z=\omega b / c_{T}$ \\
\hline Compression & $\sqrt{2(1+\sigma)}$ & $\sqrt{\frac{2}{1-\sigma}}$ & $4 k b$ & $2 \sqrt{\frac{2}{1+\sigma}} z$ \\
\hline Torsion & $2 d / b$ & $\frac{8.014}{\sqrt{(1-\sigma)}}\left(\frac{d}{b}\right)$ & $I_{2} k b$ & $\frac{1}{2} I_{2}\left(\frac{b}{d}\right) z$ \\
\hline In-plane bend & $\sqrt{\frac{1+\sigma}{6}} k b$ & 1 & $\frac{1}{3}(k b)^{3}$ & $\frac{1}{3}\left(\frac{6}{1+\sigma}\right)^{3 / 4} z^{3 / 2}$ \\
\hline Flex bend & $\sqrt{\frac{1+\sigma}{6}} k d$ & $\frac{2.886}{\sqrt{(1-\sigma)}}\left(\frac{d}{b}\right)$ & $I_{1} k b$ & $I_{1}\left(\frac{6}{1+\sigma}\right)^{1 / 4}\left(\frac{b}{d}\right)^{1 / 2} z^{1 / 2}$ \\
\hline
\end{tabular}

with $K^{2}=\sqrt{\rho d / D} \omega$ as before. The torque, Eq. (184), corresponds to a force source term given by a nonzero first moment $V_{1}$ of the tangential force

$$
V_{1}=\frac{2 D b^{2} K^{2} \sqrt{1-\sigma}}{\sqrt{6}} .
$$

This gives a source term on the right-hand side of Eq. (167b) $\widetilde{V}(\zeta)=-i \zeta V_{1}$ and the source term $\tilde{M}$ in Eq. (167a) is zero. Now defining

$$
\tilde{w}_{ \pm}(\zeta)=\frac{2 b^{2} \sqrt{1-\sigma}}{\sqrt{6}} u_{ \pm}(\xi)
$$

with $\xi=\zeta / K$ from Eq. (167) we find $u_{ \pm}$satisfies

$$
\begin{aligned}
& \quad\left[1+\xi^{2}(1-\sigma)\right] u_{+}-\left[1-\xi^{2}(1-\sigma)\right] u_{-}=0, \\
& -\sqrt{1+\xi^{2}}\left[1-\xi^{2}(1-\sigma)\right] u_{+}-i \sqrt{1-\xi^{2}}\left[1+\xi^{2}(1-\sigma)\right] u_{-} \\
& =\xi
\end{aligned}
$$

Solving these equations for $u_{ \pm}$and so $\tilde{w}_{ \pm}$, substituting into Eq. (170) for the power radiated $\left(P_{\theta}\right.$ does not contribute for this mode), and normalizing by the incident power $\omega D b^{2} K^{2} \sqrt{1-\sigma} / 2 \sqrt{6}$ yields the transmission coefficient

$$
T_{0}(\omega)=k b I_{2}(\sigma),
$$

where $I_{2}$ is the integral

$$
I_{2}(\sigma)=(1-\sigma) \frac{4}{\pi} \operatorname{Im} \int_{-\infty}^{\infty}\left(u_{+}+u_{-}\right) \xi d \xi .
$$

For $\sigma=0.33$, solving Eq. (188) for $u_{ \pm}$yields

$$
I_{2}=0.6 \quad(\sigma=0.33) \text {. }
$$

\section{APPLICATIONS AND DISCUSSIONS}

The results for the long-wavelength properties in a thinplate beam of width $b$ and thickness $d$ are brought together in Table I. The second column gives the small- $k$ dispersion re- lation in terms of the propagation speed $c_{T}$ of the in-plane shear wave in a thin plate, which is the same as the shear wave speed in the bulk medium. The third column gives the frequency cutoff $\omega_{1}$ of the lowest waveguide mode with the same transverse parity symmetry as the acoustic mode (this would be $2 \Delta$ in the scalar model). The fourth column expresses the small $\omega, k$ energy transmission coefficient $\mathcal{T}_{o}$ of the acoustic mode in terms of the wave number $k$ in the bridge and the width $b$ of the bridge. This is useful in considering the $Q$ of the fundamental vibration modes of the beam for which $k$ is of order $\pi / L$ with $L$ the length of the beam. The quantities $I_{1}$ and $I_{2}$ are Poisson-ratio-dependent numbers defined by Eqs. (182) and (190) and take on the values $I_{1}=2.3$ and $I_{2}=0.6$ for $\sigma=\frac{1}{3}$. Finally, the fifth column reexpresses the small $\omega, k$ dependence of the energy transmission coefficient $\mathcal{T}_{o}$ in terms of the frequency $\omega$. This form is particularly useful to estimate the reduction from the universal thermal conductance at low temperatures due to the strong scattering of the long-wavelength modes by an abrupt junction.

\section{A. Implications for heat transport}

The low-frequency asymptotic limit of the transmission coefficient $\mathcal{T}_{o}(\omega \rightarrow 0)$ allows us to calculate the lowtemperature variation of the thermal conductivity. If we write the dependence as

$$
\mathcal{T}_{o}(\omega \rightarrow 0)=A\left(\omega b / c_{T}\right)^{p}
$$

then relative to the universal low-temperature one-mode value $K /\left.T\right|_{u}=\pi^{2} k_{B}^{2} / 3 h$ we have for each acoustic mode

$$
\frac{K / T}{K /\left.T\right|_{u}}=A\left(\frac{k_{B} T b}{\hbar c_{T}}\right)^{p} \frac{3}{\pi^{2}} \int_{0}^{\infty} \frac{x^{2+p} e^{x}}{\left(e^{x}-1\right)^{2}} d x,
$$

where the prefactor $A$ for each mode can be found from Table I. The integral is just some $p$-dependent constant. Using the expressions in Table I the result can be written in terms of the frequency $\omega_{1}$ cutoff of the first waveguide mode of the corresponding symmetry 


$$
\frac{K / T}{K /\left.T\right|_{u}}=B\left(\frac{k_{B} T}{\hbar \omega_{1}}\right)^{p}
$$

The prefactor $B$ is a numerical constant that depends on the Poisson ratio $\sigma$, but not on geometrical factors such as $d / b$. Since the thermal excitation of the waveguide modes occurs for $k_{B} T \gtrsim 0.2 \times \hbar \omega_{1}$ (cf. the plot for the scalar model in Fig. 8) this expression indicates to what degree the plateau in $K / T$ becomes apparent as the temperature is lowered and the waveguide mode freezes out, before the reduced transmission coefficient at small frequencies begins to lower $K / T$ to zero. The ideal low-temperature universal value of $K / T$ will be more evident for smaller powers $p$. (As an example of this consider the comparison of the scalar results in Fig. 8: the solid curve shows $K / T$ for the boundary conditions leading to $p=1$, whereas the short-dashed curve is the results for boundary conditions leading to $p=3$.) This suggests that the compression and torsion modes will give contributions to $K / T$ curves similar to the result for the stress-free scalar model in Fig. 8, without a well-defined plateau at low temperatures (all have $p=1$ ), and the in-plane bend mode ( $p$ $=\frac{3}{2}$ ) will probably have no indication of a plateau. On the other hand for the flexural-bend mode $\left(p=\frac{1}{2}\right)$ the transmission coefficient increases more rapidly with increasing frequency $\mathcal{T}_{o} \sim \sqrt{\omega / \omega_{1}}$. This will lead to a more rapid increase in $K / T$ towards the universal value before significant excitation of additional modes occurs at $T \sim \hbar \omega_{1} / k_{B}$, leading to a more pronounced plateau. It should be noted that for this mode the plateau in $K / T$ only develops at very low temperatures in the thin-plate limit, reduced from the simple estimate $\hbar c_{T} / k_{B} b$ by the ratio $d / b$ of the thickness to the width of the beam.

\section{B. Implications for $Q$}

Using the third column of the table and Eq. (10) for $Q$ we get the simple estimates $Q \sim L / b$ for the fundamental compression mode, torsion mode, and flexural-bending mode, and $Q \sim(L / b)^{3}$ for the in-plane bending mode. Only for the in-plane bending mode is the isolation of the bridge modes from the supports sufficiently strong to give a large $Q$ for accessible geometries (e.g., $L / b<100)$. The strong isolation of this mode is easy to understand physically: the wide supports are very rigid against bending motion in the plane.

In many experiments on mesoscopic oscillators, modes other than the in-plane bending modes are used, and values of $Q$ significantly higher than the value suggested by the geometric ration $L / b$ are obtained. One way this is done is to use more complicated geometries, such as compound torsional oscillators arranged so that the amplitude of vibration in the bridge supports is reduced. Also, in oscillators at larger scales it is relatively easy to produce more rigid supports, for example by fabricating a bridge or cantilever making an abrupt junction to a three-dimensional support, which can also be of a different elastic material, both of which will reduce the coupling to the support modes. In mesoscopic oscillators, where the geometry is typically etched out of a single material, and undercutting by the etch comprises attempts to make an abrupt junction to a three-dimensional support, our estimates of the coupling will be more appropriate.

Our estimates of $Q$ suppose that all the energy communicated to the support modes is lost from the energy of the oscillator. This is not necessarily the case, for example, if the support material is also of sufficiently low loss and isolated from the rest of the experiment. However, our results do show that when the bridge-support coupling is large, it is important to consider the dissipation properties of the support structures as well as the bridge, cantilever, or other oscillator that is the obvious focus of attention.

\section{ACKNOWLEDGMENTS}

This work was supported by the National Science Foundation under Grant No. DMR-9873573, DARPA MTO/ MEMS under Grant No. DABT63-98-0012, and Grant No. 1999458 from the U.S.-Israel Binational Science Foundation (BSF). We thank Michael Roukes, Andrew Cleland, and Keith Schwab for many useful discussions, and Deborah Santamore for carefully reading of the manuscript.
${ }^{1}$ D. Angelescu, M. Cross, and M. Roukes, Superlattices Microstruct. 23, 673 (1998).

${ }^{2}$ L. Rego and G. Kirczenow, Phys. Rev. Lett. 81, 232 (1998).

${ }^{3}$ M.P. Blencowe, Phys. Rev. B 59, 4992 (1999).

${ }^{4}$ R. Landauer, IBM J. Res. Dev. 1, 223 (1957).

${ }^{5}$ K. Schwab, E. Henriksen, J. Worlock, and M.L. Roukes, Nature (London) 404, 974 (2000).

${ }^{6}$ R. Mihailovich and J. Parpia, Phys. Rev. Lett. 68, 3052 (1992).

${ }^{7}$ D.S. Greywall, B. Yurke, P.A. Busch, and S.C. Arney, Europhys. Lett. 34, 37 (1996).

${ }^{8}$ D. Carr and H. Craighead, J. Va. Sci. Technol. B 15, 2760 (1997).

${ }^{9}$ D. Carr, S. Evoy, L. Sekaric, H. Craighead, and J. Parpia, Appl. Phys. Lett. 75, 920 (1999).

${ }^{10}$ D. Harrington, P. Mohanty, and M. Roukes, Physica B 15, 2145 (2000)
${ }^{11}$ P. Mohanty, D. Harrington, K. Ekinci, Y. Yang, M. Murphy, and M. Roukes (unpublished).

${ }^{12}$ T. Tighe, J. Worlock, and M. Roukes, Appl. Phys. Lett. 70, 2687 (1997).

${ }^{13}$ A. Cleland and M. Roukes, Appl. Phys. Lett. 69, 2653 (1996).

${ }^{14}$ A. Szafer and A. Stone, Phys. Rev. Lett. 62, 300 (1989).

${ }^{15}$ D.H. Santamore and M.C. Cross, Phys. Rev. Lett. (to be published).

${ }^{16}$ L. Landau and E. Lifshitz, Theory of Elasticity, 3rd ed. (Butterworth-Heinemann, Oxford, 1986).

${ }^{17}$ G. Miller and H. Pursey, Proc. R. Soc. London, Ser. A 223, 521 (1954).

${ }^{18}$ K. Graff, Wave Motion in Elastic Solids (Dover, New York, 1991).

${ }^{19}$ S. Timoshenko, Theory of Elastic Stability (McGraw-Hill, New York, 1961). 
${ }^{20}$ The Rayleigh-Lamb equations usually occur in the somewhat different context of the analysis of the modes in a plate which is considered infinite in the $x y$ plane, with propagation in the $x$ direction and no dependence on the $y$ coordinate. The wave numbers $\chi_{T, L}$ then give the variation across the thickness of the plate. The equations take the same form, with the wave speed ratio $r$ given by the expression for two-dimensional elasticity theory, $r=\sqrt{2(1-\sigma)) /(1-2 \sigma)}$. For example Rego and Kircze- now (Ref. 2) plot dispersion curves for these modes across the thickness of an infinite plate.

${ }^{21}$ The sign convention for the moments $M_{x}, M_{y}$ is that $M_{i}$ is positive if it tends to produce compression in the negative $z$ side of the plate. The angular displacements $\theta_{x}, \theta_{y}$ are defined with the same convention. This is the usual definition in the elasticity literature (Ref. 18). 